\title{
On the Folding Mechanics of Square Columns with Double-Surfaced Gradients
}

\author{
Junxian Zhou $\mathbb{D}^{1},{ }^{1}$ Ruixian Qin $\mathbb{D}^{1},{ }^{1}$ and Bingzhi Chen $\mathbb{D}^{2}$ \\ ${ }^{1}$ School of Mechanical Engineering, Dalian Jiaotong University, Dalian 116028, Liaoning, China \\ ${ }^{2}$ School of Transportation and Transportation Engineering, Dalian Jiaotong University, Dalian 116028, Liaoning, China
}

Correspondence should be addressed to Bingzhi Chen; chenbingzhi06@hotmail.com

Received 15 February 2019; Revised 18 April 2019; Accepted 21 April 2019; Published 9 May 2019

Academic Editor: Michele Guida

Copyright (c) 2019 Junxian Zhou et al. This is an open access article distributed under the Creative Commons Attribution License, which permits unrestricted use, distribution, and reproduction in any medium, provided the original work is properly cited.

The implementation of graded thicknesses in structural designs is an efficient strategy for improving the energy absorption performance of thin-walled columns. In this study, the mechanical responses of square columns with double-surfaced gradients under axial crushing forces were analytically and numerically investigated. A mathematical expression for the mean crushing force on a novel tube design is derived according to the super folding element method by introducing two novel parameters: the average thickness of the efficient energy absorbing region (EEAR) and global average thickness. The analytical predictions show good agreement with the simulation results that were obtained using the nonlinear finite element program ANSYS/LS-DYNA. The results demonstrate that a greater coefficient of average thickness of the EEAR versus global average thickness improves the energy absorption efficiency. However, increasing this coefficient largely depends on the sectional material distribution law. To explore the effects of this law on crushing responses, three different material distribution types were investigated. A significant improvement in specific energy absorption (approximately $30 \%$ ) was obtained for a double-surfaced column with an optimized material distribution type compared to that of a square column with a uniform thickness.

\section{Introduction}

Based on a greater emphasis on the passive safety of transportation, which includes the security of passengers and vital equipment, the performance requirements of energy absorption devices have increased significantly. An energy absorption device typically consists of a thin-walled structure with a steady and foreseeable collapse mode, high manufacturability, and moderate cost $[1,2]$.

Additionally, the implementation of lightweight designs is important for energy conservation. Therefore, novel structures with high specific energy absorption (SEA) are required. The energy absorption of a thin-walled tube is closely related to its section configuration and crushing mode. Accordingly, research on the crushing behaviors of metal tubes with various cross-sectional patterns has received significant attention. Alexander [3] utilized experimental data to develop an approximate mathematical model of the concertina crushing mode to estimate the mechanical responses of cylindrical tubes subjected to quasi-static axial loads. However, depending on the diameter-to-wall-thickness ratio $(D / t)$ and lengthto-diameter ratio $(L / D)$, a cylindrical column may be crushed not only in the concertina mode, but also in diamond and mixed modes [4], which were not considered in the theoretical model [3]. Many researchers have investigated the concertina and diamond modes [4-7] and developed improved theoretical models for predicting the folding patterns and mean crushing loads of cylindrical tubes.

Similarly, crushing modes of square tubes are closely related to the cross-sectional width-to-wall-thickness ratio $(B / t)$ and length-to-cross-sectional-width ratio $(L / B)$; such relationships are summarized in a graph proposed by Abramowicz and Jones [8], which was constructed based on several experiments. Through theoretical research, Wierzbicki and Abramowicz [9] modeled the basic folding mechanism of a square tube with a quasi-inextensional collapse mode and referred to their findings as the super folding element theory. In this theory, a quasi-inextensional 
basic folding element was established. This element consists of trapezoidal, horizontal cylindrical, conical, and toroidal surfaces. Three energy-dissipating approaches-folding along stationary plastic hinge lines, rolling at inclined plastic hinge lines, and quasi-inextensional deformation in the toroidal area-were considered for the element. The inclined hinge lines and toroidal surface were essentially confined to the corner regions. Therefore, it is believed that the corner regions contribute significantly to energy absorption.

Consequently, a strategy that increases the number of corners (polygonal columns) [10-13] has been adopted to improve the energy absorption efficiency. Yamashita et al. [11] found that the buckling mode and dynamic performance of polygonal tubes are affected by the angles between neighboring flanges. Additionally, angles in the range of $90-120^{\circ}$ are the most reasonable angles for achieving the highest mean load. However, designs based on polygonal cross sections have a finite amount of energy absorption improvement because the additional side walls lead to corner angles exceeding the reasonable range $\left(90-120^{\circ}\right)$.

The problem of increasing the number of corners while maintaining corner angles in the range of $90-120^{\circ}$ can be solved through the use of tubes with novel cross sections, known as a non-convex multi-corner (NCMC) columns, which were developed by Tang and Liu et al. [14, 15]. Compared to traditional rectangular structures, a significant improvement in SEA can be obtained by using these novel columns. Similarly, an improvement in SEA can also be achieved by using another multi-corner structure, known as a star-shaped column, which was proposed by Fan et al. [16, 17].

Based on previous studies [14-17], a potential deficiency of NCMC and star-shaped tubes has been identified. Specifically, the crushing mode will deform into a noncompact mode as the tubes become thicker. Inspired by the structure of bamboo, Liu et al. [15] inserted perforated plates into NCMC tubes to counteract this deficiency. Additionally, Liu et al. [17] placed two polygonal shells on the inside and outside of a starshaped column to avoid irregular folding patterns.

More recently, some researchers put their attention on graded structures. Baykasoglu and Cetin studied crashworthiness of circular tubes with gradient thicknesses [18]. The crushing resistances of circular tubes with axially graded thickness were then explored by $\mathrm{Xu}$ et al. [19]. Graded thicknesses in the longitudinal direction were also extended to square [20] and multicell columns [21]; and corresponding performance optimization was presented. In addition, Zhang et al. investigated crushing resistances of non-uniformthickness conical tubes [22] and bending collapse behaviors of square tubes with variable thickness [23]; they also summarized state of the art of energy absorption of graded structures and materials [24]. These studies have proved that it is a significant approach to improve the energy absorption performance by changing the material distribution of thinwalled structures. In addition, with advancements in material rolling technology, metal plates with graded thickness could be manufactured without difficulty or high costs. Accordingly, such tubes can be manufactured using tailor-rolledblank technology (TRB), which is also used to produce lightweight vehicle components efficiently $[25,26]$.
Similarly, Zhang et al. [27] improved the energy absorption performance by optimizing the cross-sectional material distribution of square tubes. This approach is similar to the strategy of increasing the number of corners. It considers the plastic energy to be dissipated centrally in the corner regions, as reported in [9]. Therefore, this approach concentrates more material in the corner regions and improves the energy absorption performance of square tubes.

Research [27] shows two material distribution methods for the cross sections of square tubes, namely, the single surface gradient (SSG) and double-surfaced gradient (DSG) methods; collapse modes and crashworthiness were experimentally and numerically investigated under axial loading. Compared to a traditional square tube, a maximum improvement of $27 \%$ in terms of specific energy absorption was obtained by the DSG square tube.

However, the energy absorption mechanism of the DSG tube is still unknown and the influence of cross-sectional material distribution on energy absorption is unclear. In this study, the crushing mechanism of a DSG square tube was theoretically analyzed based on the super folding element method to predict energy dissipation of the tube. A mathematical model was developed for the mean crushing force. Additionally, simulations were conducted to validate the accuracy of the theoretical predictions and investigate the influence of key variables of the mathematical model on the mechanical responses using the nonlinear finite element software ANSYS/LS-DYNA. Finally, the law of crosssectional material distribution, which can effectively improve energy absorption, was investigated.

\section{Material Distribution Types}

Zhang et al. [27] proposed two material distribution types for square tubes, namely, the SSG and DSG, as shown in Figure 1. This figure illustrates that the cross-sectional shapes are defined by three parameters: the thickness of the middle regions $(t 1)$, corner thickness $(t 2)$, and cross-sectional width $(B)$. The authors tested numerous specimens under quasistatic axial impact conditions using two types of gradient thicknesses. These tests were conducted to investigate the effects of the two types (SSG and DSG) on folding patterns and crashworthiness performance. According to [27], despite maintaining consistent values for all the specimens ( $B$ was maintained at $36 \mathrm{~mm}$, $t 1$ ranged from 0.6 to 1.2 , and $t 2$ ranged from 1.2 to 1.8 for all tubes), there were still significant differences between the mechanical responses of the SSG and DSG tubes.

First, the folding wavelength and lobes of the SSG square tubes gradually increased with a thickening of the corner regions. Consequently, with the maximum thickness gradient, irregular deformations occurred in the thinnest regions of the SSG square column. For the DSG square columns, amplification of the folding lobes and folding wavelength was not noticeable and irregular deformation did not occur, even with the same thickness gradient as that of the SSG tube mentioned previously. Second, based on the longer wavelength, the force-displacement curves of the SSG square columns fluctuated significantly more than those of the DSG 


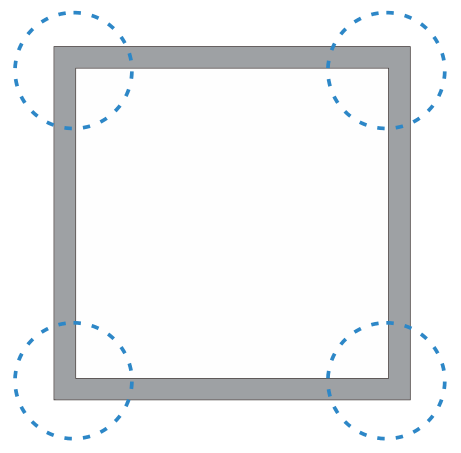

SQU
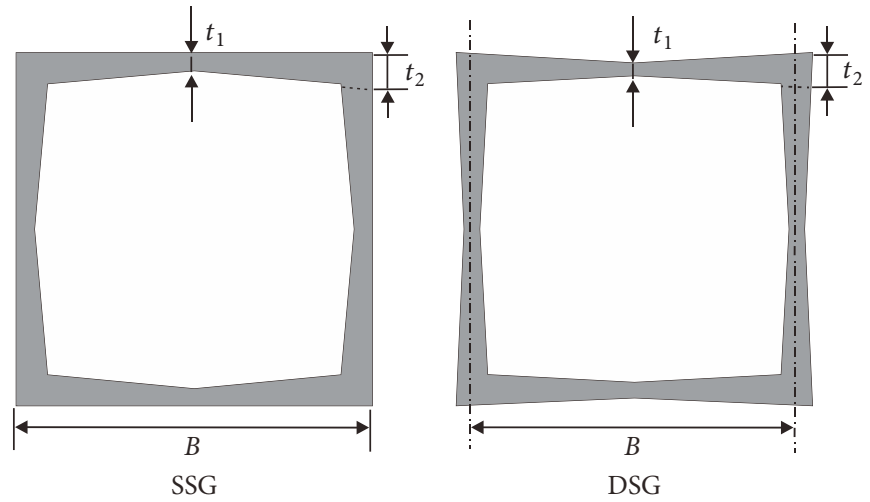

FIGURE 1: Material distributions in the various types of square columns.

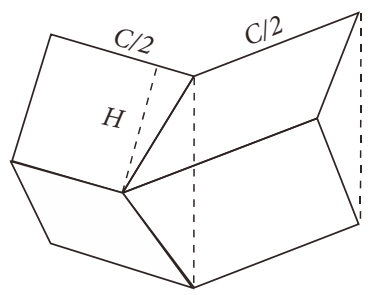

(a)

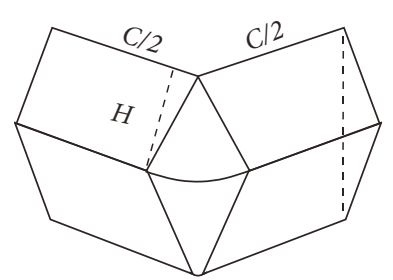

(b)

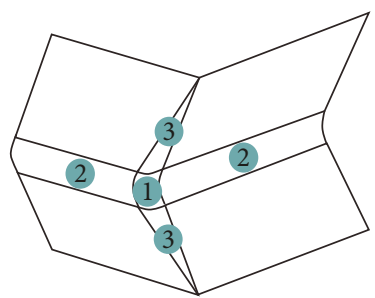

(c)

Figure 2: Simplified collapse mechanisms of the two folding elements: (a) Type I and (b) Type II; and (c) deformation areas of the Type I element.

square columns and the distances between neighboring peaks in the curves were much greater than those of the DSG structures. Finally, compared to a traditional square column, the mean crushing load of the DSG square column with the maximum graded thickness increased by $35.5 \%$, which is greater than the increase provided by the SSG square tube with the same graded thickness.

To sum up, this study focused on the theoretical analysis of the folding mechanism and energy absorption enhancement of DSG tubes.

\section{Theoretical Analysis of a Square Column with a DSG}

An analytical solution for the mechanical responses of square tubes under axial progressive loading was derived by Wierzbicki and Abramowicz through the introduction of the coefficient of effective crushing distance [9].

According to their study, a modified expression for the mean static impact force $P_{m}$ can be defined as shown in

$$
P_{m}=13.06 \sigma_{0} B^{1 / 3} t^{5 / 3}
$$

where $\sigma_{0}$ is the equivalent flow stress and $B$ and $t$ are the sectional width and wall thickness, respectively.

The expression for the half folding wavelength $H$ is

$$
H=1.27 B^{2 / 3} t^{1 / 3} .
$$

Considering strain hardening effects, the equivalent material flow stress with power law hardening behavior can be calculated as follows [28]:

$$
\sigma_{0}=\sqrt{\frac{\sigma_{y} \sigma_{u}}{1+n}},
$$

where $\sigma_{y}$ denotes the initial yield strength and $\sigma_{u}$ and $n$ are the ultimate strength and power law exponent, respectively.

3.1. Basic Collapse Elements. As shown in Figure 2, two basic folding elements for the theoretical analysis of rectangular tube crushing responses are considered in the super folding element method: Type I, which was studied by Wierzbicki and Abramowicz [9], and Type II, which was investigated in [29-31]. In these studies, the collapse mechanisms of the two basic folding elements were accurately described using a rigorous kinematic approach. Additionally, the energy dissipation of the two elements was precisely analyzed using four energy dissipating contributors: the horizontal plastic hinge line, inclined plastic hinge line, toroidal surface, and conical surface. Therefore, these two basic folding elements can be used to predict the impact responses of columns with different cross-sectional shapes $[9,14,15,32,33]$.

The energy dissipation of Type I, which is illustrated in Figure 2(a), can be estimated using the following equation [9].

$$
E_{\text {type } 1}=M_{0}\left(\frac{16 I_{1} H b}{t}+2 \pi C+\frac{4 I_{3} H^{2}}{b}\right),
$$




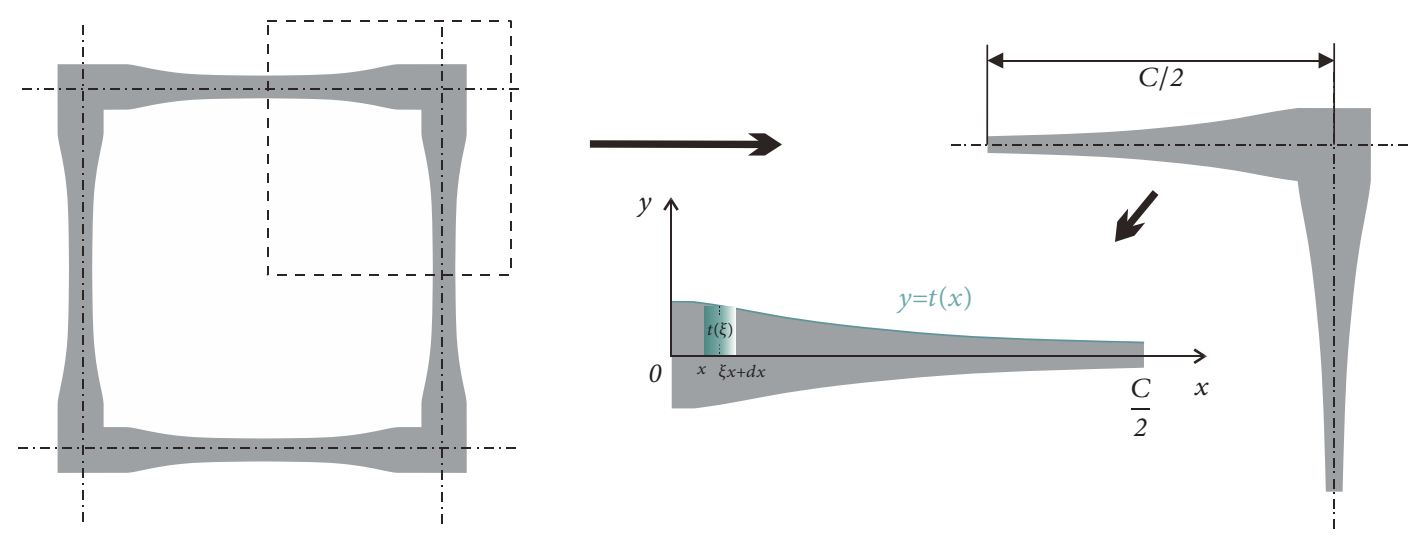

FIGURE 3: Cross section of a DSG square tube.

where $I_{1}$ and $I_{3}$ are 0.58 and 1.11 (for square tubes), respectively, $C$ denotes the flange length of a corner, and $b$ denotes the smaller radius of the toroidal surface [9]. Similarly, the energy absorption formula for the Type II folding element, which is illustrated in Figure 2(b), was deduced by Abramowicz and John [29] and defined as follows:

$$
E_{\text {type } 2}=M_{0}\left(\frac{8 H^{2}}{t}+2 \pi C+4 H\right) \text {. }
$$

This energy dissipation expression was modified by introducing an improved deformation mechanism [30] and rewritten as follows:

$$
E_{\text {type2 }}=M_{0}\left(\frac{2 \pi H^{2}}{t}+2 \pi C+\pi H\right) .
$$

3.2. Energy Dissipation of Type I Folding Element with a DSG. This study focused on the quasi-inextensional mode, which consists of four Type I elements subjected to a single wavelength crushing. In accordance with the super folding element theory [9], the collapse mechanism of the Type I folding element is illustrated in Figure 2(c). In a complete collapse, energy dissipation is derived from three main contributors [9]: the horizontal plastic hinge lines (see Figure 2(c), area 2), toroidal surface (see Figure 2(c), area 1), and inclined plastic hinge lines (see Figure 2(c), area 3).

Considering a Type I folding element with a uniform thickness, the dissipated energy at the horizontal plastic hinge lines can be expressed as follows [9]:

$$
E_{2}=2 \pi M_{0} C
$$

where $M_{0}$ is the plastic limit moment $\left(M_{0}=1 / 4 \sigma_{0} t^{2}\right)$ and $C$ represents the flange length around the corner. Therefore, obtaining an accurate expression for the plastic limit moment of a DSG Type I element with varying thickness is relatively complicated.

In this study, the bending energy dissipated by a DSG plate with a nonuniform thickness was approximated using an integration method. This ensured that the theoretical analysis was efficient and the final mean load expression had a concise form. Subsequently, as shown in Figure 3, a two-dimensional coordinate system was established on a DSG plate with a flange length of $C / 2$. The vertical $y$-axis represents the thickness and the horizontal $\mathrm{x}$-axis represents the flange length. As illustrated in Figure 3, the origin of the coordinate system is located at the end of the plate midline. $t(x)$ is a continuous thickness function over the length interval $[0, C / 2]$ and can be fitted according to the geometric configuration of the DSG plate. Accordingly, the bending energy of an infinitesimal plate with a width of $d x$ can be expressed as follows:

$$
d E=\frac{1}{2} \sigma_{0} \pi t^{2}(\xi) d x,
$$

where $\xi$ is an arbitrary point within the interval $[x, x+d x]$. Furthermore, the bending energy of a DSG folding element with a flange length of $C$ can be calculated as follows:

$$
E_{2}^{D S G}=2 \int_{0}^{C / 2} \frac{1}{2} \sigma_{0} \pi[2 t(x)]^{2} d x=2 C \sigma_{0} \pi t^{2}(\zeta)
$$

where $\zeta$ denotes a point within the interval $[0, C / 2]$. To facilitate theoretical analysis, a new parameter, namely, the global average thickness $\left(t_{a}\right)$, was introduced. This parameter can be expressed as follows:

$$
t_{a} \approx 2 t(\zeta)
$$

Using (10), (9) can then be simplified to produce (11) as follows:

$$
E_{2}^{D S G} \approx \frac{1}{2} \sigma_{0} \pi C t_{a}^{2}
$$

Based on the uncomplicated nature of the thickness functions for DSG square tubes, the parameter $t_{a}$ can be calculated easily.

It is worth noting that when the tube length and width are constant, the change in the average thickness determines the mass of the tube.

The remaining energy absorbed by the Type I folding element stems from two efficient contributors [9]: the inclined plastic hinge lines and toroidal surface. The region 


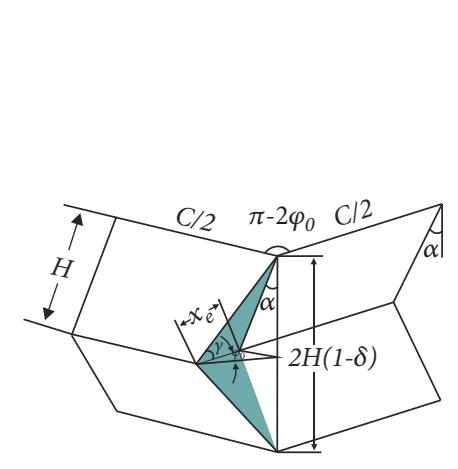

(a)

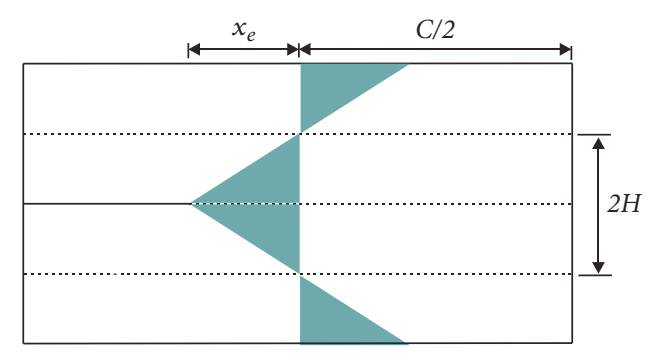

(b)

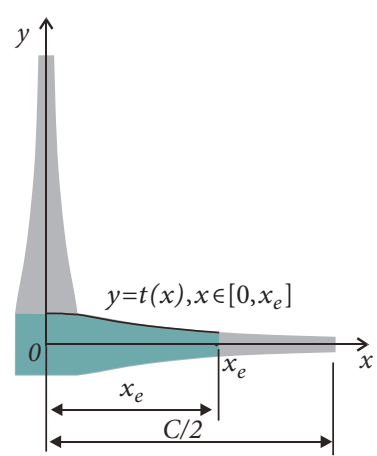

(c)

Figure 4: (a) Geometry of the Type I basic folding element, (b) triangular simplified regions representing the EEAR, and (c) cross section of the Type I element with a DSG.

participating in the energy absorption by these contributors (i.e., the efficient energy absorbing region (EEAR)) is largely focused near the corners, as shown by the blue triangles in Figures 4(a) and 4(b). The energy dissipated by the EEAR in a Type I folding element with constant thickness can be obtained by integrating the two contributors as follows [9]:

$$
E_{1}+E_{3}=M_{0}\left(16 \frac{H b}{t} I_{1}+4 \frac{H^{2}}{b} I_{3}\right)
$$

For the DSG Type I element, deriving a rigorous expression for the energy dissipated by the two contributors is relatively difficult. Therefore, the method used for handling the horizontal plastic hinge lines in the DSG section was also adopted for this purpose. The energy contribution of the inclined plastic hinge lines and toroidal surface in the DSG Type I element can be calculated using the following equations.

$$
\begin{aligned}
E_{1}^{D S G} & =\frac{\int_{0}^{x_{e}} 4 \sigma_{0}[2 t(x)]^{2}(H b / 2 t(x)) I_{1} d x}{x_{e}} \\
& =4 \sigma_{0}[2 t(\eta)]^{2} \frac{H b}{2 t(\eta)} I_{1} \approx 4 \sigma_{0} t_{e}{ }^{2} \frac{H b}{t_{e}} I_{1}, \\
E_{3}^{D S G} & =\frac{\int_{0}^{x_{e}} \sigma_{0}[2 t(x)]^{2}\left(H^{2} / b\right) I_{3} d x}{x_{e}} \\
& =\sigma_{0}[2 t(\eta)]^{2} \frac{H^{2}}{b} I_{3} \approx \sigma_{0} t_{e}{ }^{2} \frac{H^{2}}{b} I_{3}, \\
2 t(\eta) & \approx t_{e},
\end{aligned}
$$

where $x_{e}$ denotes the width of the EEAR, as shown in Figures $4(\mathrm{a})-4(\mathrm{c}), t(x)$ is the thickness function, and $\eta$ denotes a point within the interval $\left[0, x_{e}\right]$. The parameter $t_{e}$ is the average thickness of the EEAR, which can be calculated as follows:

$$
t_{e}=\frac{2 \int_{0}^{x_{e}} t(x) d x}{x_{e}} .
$$

Therefore, the primary challenge in solving for $t_{e}$ is determining the width of the EEAR (i.e., $x_{e}$, where $x_{e}$ is equal to the base-side length of the blue triangles shown in Figure 4(a)). In this figure, the initial geometry of the folding element is defined by the height $2 \mathrm{H}$, total width $\mathrm{C}$, and angle $\pi-2 \varphi_{0}$ between two neighboring plates. The geometry in the current configuration can be described by the angle of rotation of the side panels $\alpha$ or the crushing distance $2 H \delta$, where $\delta$ denotes the crushing distance coefficient. The other angle $\gamma$ is related to $\varphi_{0}$ and $\alpha$ as follows [9]:

$$
\begin{aligned}
\gamma & =\tan ^{-1}\left(\frac{\tan \varphi_{0}}{\sin \alpha}\right), \\
\varphi_{0} & =\frac{\pi}{4} .
\end{aligned}
$$

Based on the geometric characteristics of the Type I folding element, the parameter $x_{e}$ can be calculated using the following equations.

$$
\begin{gathered}
\alpha=\cos ^{-1} \frac{H(1-\delta)}{H}=\cos ^{-1}(1-\delta), \\
x_{e}=\sin \alpha H=\varepsilon H ; \quad \varepsilon=\sqrt{1-(1-\delta)^{2}},
\end{gathered}
$$

where $\varepsilon$ is the width coefficient of the EEAR. Therefore, for a Type I element that experiences complete compression with a crushing distance coefficient of $\delta=1, x_{e}=H$. However, based on the investigation conducted by Wierzbicki and Abramowicz [9], the actual crushing distance coefficient $\delta$ is approximately $0.7-0.75$. Therefore, theoretically, the width coefficient of the EEAR $\varepsilon$ is approximately $0.95-0.97$. In the following analysis, the two coefficients $\delta$ and $\varepsilon$ were set to 0.70 and 0.95 , respectively.

3.3. Mean Crushing Force. As mentioned above, the energy dissipation of a square column stems from three main contributors (horizontal plastic hinge lines, inclined plastic hinge lines, and the toroidal surface). This assumption is also appropriate for the DSG square column. Therefore, equating the external work to the internal energy absorption of the 
DSG structure in one wavelength period yields the following equation.

$$
\begin{aligned}
2 H \delta P_{m} & =4\left(E_{1}^{D S G}+E_{2}^{D S G}+E_{3}^{D S G}\right) \\
& =\frac{1}{4} \sigma_{0}\left(64 I_{1} t_{e}^{2} \frac{b H}{t_{e}}+16 I_{3} t_{e}^{2} \frac{H^{2}}{b}+8 t_{a}^{2} \pi C\right) .
\end{aligned}
$$

Considering that $L_{C}$ is the section perimeter, (20) can be simplified to produce (21) as follows:

$$
\frac{P_{m}}{(1 / 4) \sigma_{0}}=\frac{1}{\delta}\left\{t_{e}^{2}\left(32 I_{1} \frac{b}{t_{e}}+8 I_{3} \frac{H}{b}\right)+t_{a}^{2} \pi \frac{L_{C}}{H}\right\} .
$$

Minimizing (21) with respect to the unknown parameters $H$ and $b$ yields the following equations.

$$
\begin{aligned}
& \frac{\partial P_{m}}{\partial H}=0: \quad 8 I_{3} t_{e}^{3} \frac{1}{b / t_{e}}=\pi t_{a}^{2} \frac{L_{C}}{\left(H / t_{e}\right)^{2}} \\
& \frac{\partial P_{m}}{\partial b}=0: \quad \frac{4 I_{1}}{I_{3}}\left(\frac{b}{t_{e}}\right)^{2}=\frac{H}{t_{e}}
\end{aligned}
$$

The two significant parameters $b$ and $H$ can be obtained by solving (22) and (23) as follows:

$$
\begin{gathered}
b=A t_{e}\left(\frac{t_{a}^{2} L_{C}}{t_{e}^{3}}\right)^{1 / 3}, \\
H=B t_{e}\left(\frac{t_{a}^{2} L_{C}}{t_{e}^{3}}\right)^{2 / 3},
\end{gathered}
$$

where

$$
\begin{aligned}
& A=\left(\frac{\pi I_{3}}{128 I_{1}^{2}}\right)^{1 / 3}, \\
& B=\frac{4 I_{1}}{I_{3}}\left(\frac{\pi I_{3}}{128 I_{1}^{2}}\right)^{2 / 3} .
\end{aligned}
$$

Through the substitution of (24) and (25) into (21), the mean crushing force can be obtained as follows:

$$
\begin{aligned}
& \frac{P_{m}}{(1 / 4) \sigma_{0}}=\frac{1}{\delta}\left\{32 A I_{1} t_{e}^{2}\left(\frac{t_{a}^{2} L_{C}}{t_{e}^{3}}\right)^{1 / 3}\right. \\
& \left.+\frac{\pi L_{C} t_{a}^{2}}{B t_{e}}\left(\frac{t_{e}^{3}}{t_{a}^{2} L_{C}}\right)^{2 / 3}+\frac{8 I_{3} B t_{e}^{2}}{A}\left(\frac{t_{a}^{2} L_{C}}{t_{e}^{3}}\right)^{1 / 3}\right\} .
\end{aligned}
$$

Equation (28) can then be simplified to produce the following equation.

$$
P_{m}=\frac{1}{\delta} P_{s} \sigma_{0} t_{e}\left(t_{a}^{2} L_{C}\right)^{1 / 3}
$$

where

$$
P_{s}=24\left(\frac{\pi I_{1} I_{3}}{128}\right)^{1 / 3} .
$$

For square tubes with $I_{1}=0.58$ and $I_{3}=1.11[9]$, the parameter $P_{s}$ is equal to 6.021 .

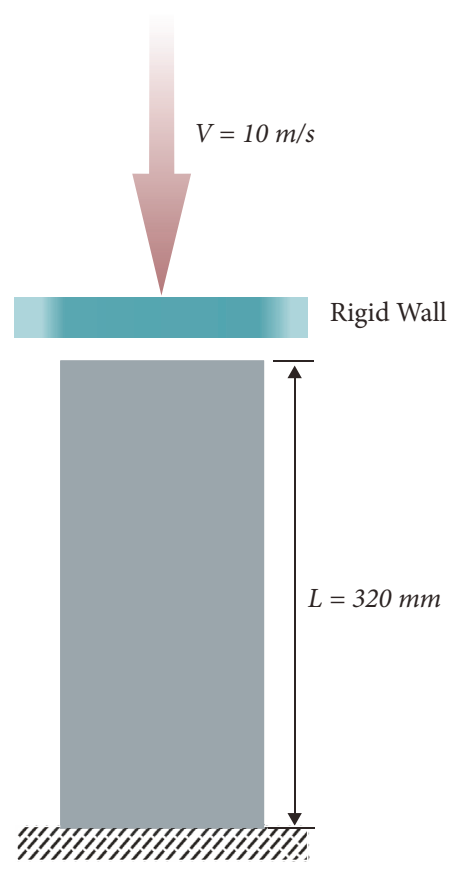

FIGURE 5: Boundary conditions for the specimens in the numerical simulations.

\section{Numerical Simulations}

The columns considered in this study were DSG square tubes and numerical simulations were conducted for the columns using the nonlinear finite element software ANSYS/LSDYNA to simulate crushing. The boundary conditions for the numerical simulations are illustrated in Figure 5. As shown in this figure, the bottom of the tube was clamped, and the top was crushed by a massive rigid wall with a constant velocity of $10 \mathrm{~m} / \mathrm{s}$ in the axial direction. Two contacts were considered in the model. Automatic single surface contact was introduced to simulate the self-contact of the column and automatic node-to-surface contact was applied between the column and rigid wall. Static and dynamic friction were included in these contacts with the same coefficient values of 0.2 . Identical materials and geometry dimensions with a length $L=320 \mathrm{~mm}$ and cross-sectional width $B=90 \mathrm{~mm}$ were used for all finite element models. To ensure a reasonable deformation mode and lower initial peak force, triggers with a depth of $0.6 \mathrm{~mm}$ were installed on the two parallel plates at a distance $H$ below the crushed end of the columns, as illustrated in Figure 6.

4.1. Numerical Models. Figure 7 presents the cross sections of the DSG square tubes with two types of material distributions. In order to intuitively reflect the effect of material distribution type on the energy absorption performance, linear thickness functions with obvious characteristics were adopted by the two types of tubes (Type 1 and Type 2). The main parameters of the sectional geometry are the maximum thickness $t_{1}$, minimum thickness $t_{2}$, and sectional width $B$. The difference between the two distribution types was the width of the uniform thickness area. In Figure 7, the uniform thickness area and gradient thickness area are denoted by 

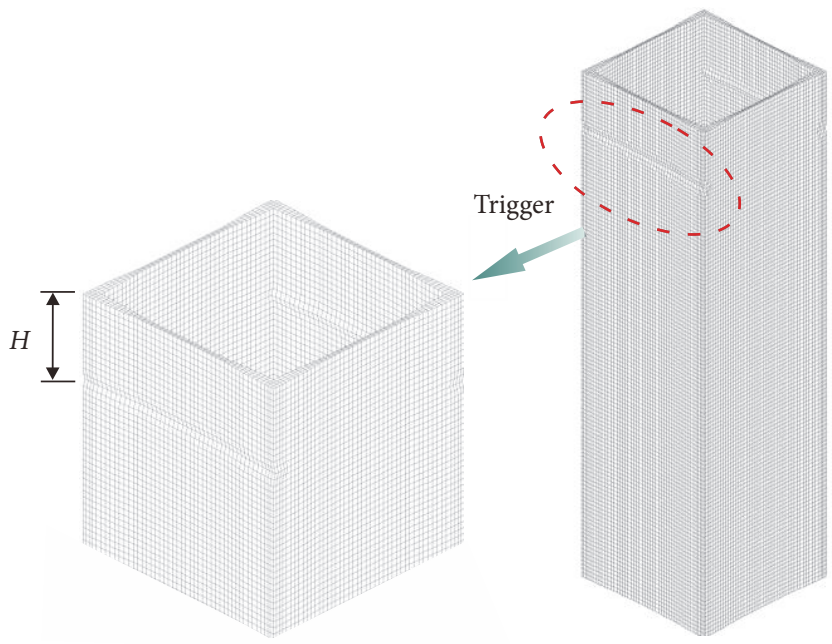

FIGURE 6: FE model of a DSG square tube with imperfections.

pink and gray, respectively. There was almost no area with a uniform thickness in the Type 1 tubes, except for the joints between adjacent plates. In contrast, the Type 2 tubes had a large uniform thickness area, which was characterized by the width $w_{e}$. To achieve higher energy absorption efficiency compared to the Type 1 DSG tubes, a strategy for increasing the amount of material in the EEAR was adopted for the Type 2 tubes. Therefore, the width of the uniform thickness area $w_{e}$ was set equal to the width of the EEAR $x_{e}$ (i.e., $w_{e}=x_{e}=$ $0.95 H)$.

The columns were modeled using solid elements. Mesh convergence analysis was conducted to determine a sufficient mesh density to obtain an accurate mechanical response. Simulations of the two representative columns with different numbers of elements along their thicknesses were performed to determine the optimal number of elements, as shown in Figure 8(a). The optimal element size was also determined, as shown in Figure 8(b). As a result, three elements along the thickness direction with a mesh size of $1 \mathrm{~mm}$ along the width and length directions were implemented to ensure sufficient mesh density and achieve accurate results.

4.2. Material Characterization. The material used in the simulations was the aluminum extrusion alloy AA6060 T4, which has the following properties [28]: Young's modulus E $=68.21 \mathrm{GPa}$, Poisson's ratio $\mu=0.3$, initial yield strength $\sigma_{y}=$ $80 \mathrm{MPa}$, ultimate strength $\sigma_{u}=173 \mathrm{MPa}$, and the power law exponent $n=0.23$. The engineering tensile-stress strain curve of the material is shown in Figure 9.

4.3. Numerical Results. The geometric parameters of the specimens are listed in Table 1. All specimens were divided into three groups: A, B, and C. Columns in each group have the same global average thickness and a constant mass, and global average thicknesses for groups A, B, and C are $3.0 \mathrm{~mm}, 3.2 \mathrm{~mm}$, and $3.4 \mathrm{~mm}$, respectively. A concise numbering method was used for the columns. For instance, A-TRA denotes a traditional group A column with a constant
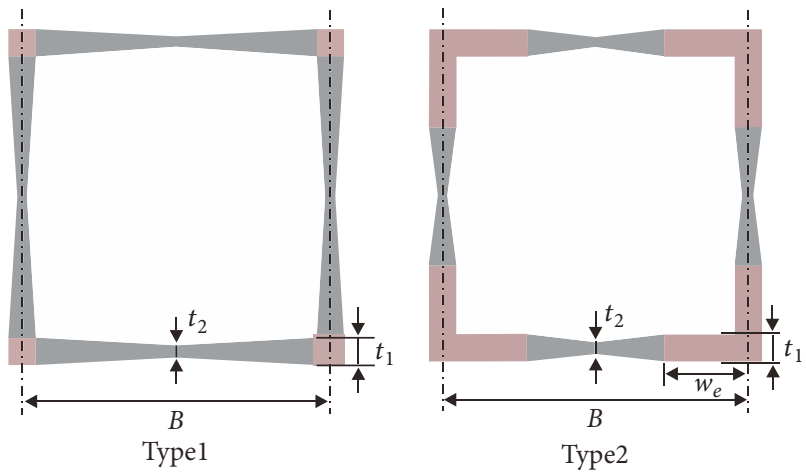

Uniform thickness area

Gradient thickness area

FIgURE 7: Two types of material distributions.

thickness of $3.0 \mathrm{~mm}$, and Type 1-A-1 denotes a DSG square tube with a Type 1 material distribution type. The final number indicates that the magnitude of the thickness gradient is equal to one. All finite element models were computed using the ANSYS/LS-DYNA software. Correspondingly, the crushing processes of all specimens are illustrated in Figure 10, which shows the folding shapes of the columns at displacements of 20 and $230 \mathrm{~mm}$.

Based on the theoretical analysis in Section 3, two parameters are essential for predicting the mean load of the DSG tube theoretically: the global average thickness of the tube $t_{a}$, which can be easily obtained based on the geometric configuration, and average thickness of the EEAR $t_{e}$, which can be determined for a known half wavelength $H$ using (25). However, calculating $H$ using (16) and (19) requires a known value of $t_{e}$, meaning the calculation process is circular.

Based on the impact responses obtained from the numerical simulations, the relationship between the half wavelength and thickness gradient of the Type 1 DSG tubes was plotted as the half wavelength versus the maximum thickness $\left(t_{1}\right)$ curves, as shown in Figure 11. The half wavelength increased slightly or remained unchanged with an increase in the thickness gradient. This trend was observed for all three average thickness conditions (Figures 11(a)-11(c)). Furthermore, the theoretical predictions (red lines in Figure 10) for the traditional square tubes agree well with the simulation results for DSG tubes with the same average thicknesses as the traditional square tubes, regardless of the thickness gradient. Therefore, the $t_{e}$ value of a DSG tube can be calculated using the theoretical half wavelength of a traditional square tube with the same average thickness as the DSG tube.

4.3.1. Verification of Theoretical Predictions. Based on the inertia and strain rate effects of the tested materials, the mean loads under dynamic loading were larger than the theoretical results predicted by (29), which were derived for quasistatic compression conditions. The dynamic amplification coefficient, which was proposed by Hanssen et al. [34] based on an experimental study of the dynamic and quasi-static responses of thin-walled columns, can be introduced to fix this discrepancy. The amplification of the aluminum is 
TABLE 1: Dimensions and physical properties of the specimens.

\begin{tabular}{|c|c|c|c|c|c|c|}
\hline Specimen & $t_{1}(\mathrm{~mm})$ & $t_{2}(\mathrm{~mm})$ & $w_{e}$ & $t_{e}(\mathrm{~mm})$ & $t_{a}(\mathrm{~mm})$ & Mass (g) \\
\hline A-TRA & 3.00 & 3.00 & 0 & 3.00 & 3.00 & 935.3 \\
\hline Type 1-A-1 & 3.20 & 2.80 & 0 & 3.08 & 3.00 & 935.3 \\
\hline Type 1-A-2 & 3.40 & 2.60 & 0 & 3.16 & 3.00 & 935.3 \\
\hline Type 1-A-3 & 3.60 & 2.40 & 0 & 3.24 & 3.00 & 935.3 \\
\hline B-TRA & 3.20 & 3.20 & 0 & 3.20 & 3.20 & 997.6 \\
\hline Type 1-B-1 & 3.40 & 3.00 & 0 & 3.28 & 3.20 & 997.6 \\
\hline Type 1-B-2 & 3.60 & 2.80 & 0 & 3.36 & 3.20 & 997.6 \\
\hline Type 1-B-3 & 3.80 & 2.60 & 0 & 3.44 & 3.20 & 997.6 \\
\hline C-TRA & 3.40 & 3.40 & 0 & 3.40 & 3.40 & 1060.5 \\
\hline Type 1-C-1 & 3.60 & 3.20 & 0 & 3.48 & 3.40 & 1060.5 \\
\hline Type 1-C-2 & 3.80 & 3.00 & 0 & 3.56 & 3.40 & 1060.5 \\
\hline Type 1-C-3 & 4.00 & 2.80 & 0 & 3.64 & 3.40 & 1060.5 \\
\hline Type 2-A-1 & 3.20 & 2.19 & $0.95 \mathrm{H}$ & 3.20 & 3.00 & 935.3 \\
\hline Type 2-A-2 & 3.40 & 1.38 & $0.95 H$ & 3.40 & 3.00 & 935.3 \\
\hline Type 2-B-1 & 3.40 & 2.36 & $0.95 \mathrm{H}$ & 3.40 & 3.20 & 997.6 \\
\hline Type 2-B-2 & 3.60 & 1.59 & $0.95 H$ & 3.60 & 3.20 & 997.6 \\
\hline Type 2-C-1 & 3.60 & 2.53 & $0.95 H$ & 3.60 & 3.40 & 1060.5 \\
\hline Type 2-C-2 & 3.80 & 1.72 & $0.95 \mathrm{H}$ & 3.80 & 3.40 & 1060.5 \\
\hline
\end{tabular}

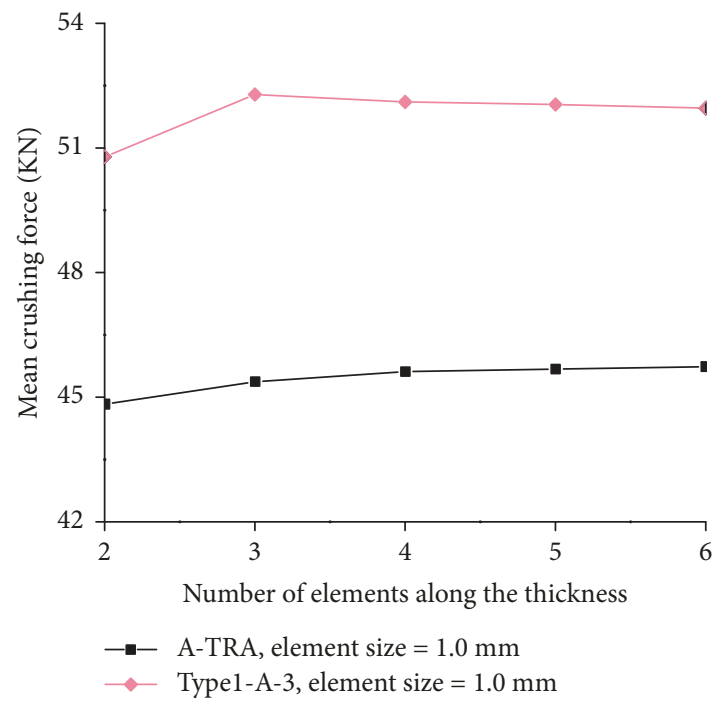

(a)

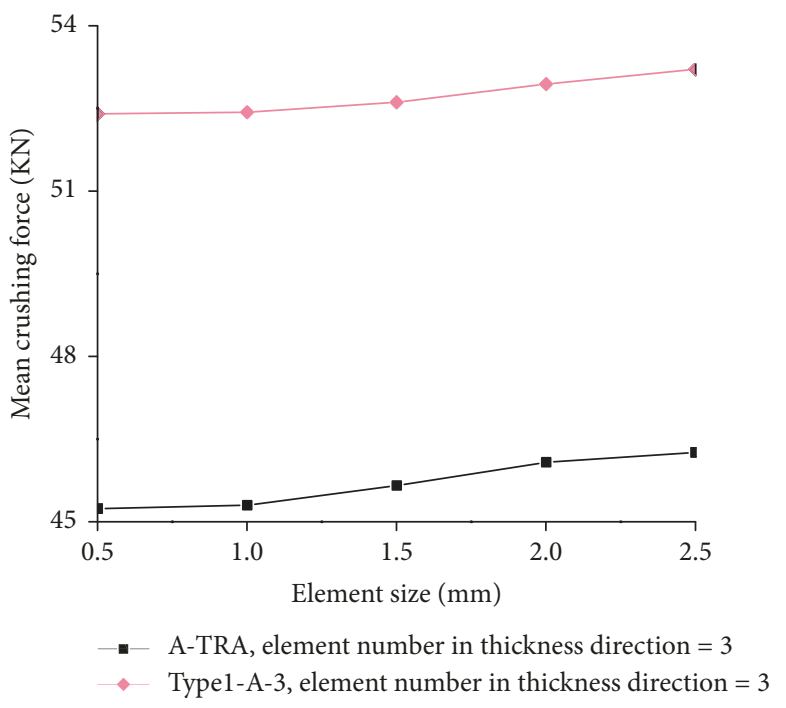

(b)

FIGURE 8: Force convergence: (a) different numbers of elements along the thickness and (b) element size.

dominated by inertial effects because it is insensitive to the strain rate. Langseth and Hopperstad [35] proposed a range of 1.3-1.6 for the coefficients of AA6060 T4 tubes with no triggers, while a lower value was proposed for tubes with triggers. In this study, to deal with the triggers, a value of 1.15 was adopted to account for the theoretical prediction of a specimen with an imperfection. Therefore, (29) was modified to create (31), which can be used to predict the crushing resistance of DSG tubes under dynamic compression.

$$
P_{m}=1.15 \times 6.021 \frac{1}{\delta} \sigma_{0} t_{e}\left(t_{a}^{2} L_{C}\right)^{1 / 3} .
$$

Using the Type 2-A-1 sample as an example, it was found that $t_{e}=3.2 \mathrm{~mm}$ and $t_{a}=3.0 \mathrm{~mm}$. By substituting these values into (31), the theoretical solution for the mean load can be expressed as

$$
\begin{aligned}
P_{m} & =1.15 \times 6.021 \times \frac{1}{0.7} \times 106 \times 3.2\left(3^{2} \times 360\right)^{1 / 3} \\
& =49.65 \times 10^{3} \mathrm{~N} .
\end{aligned}
$$

Similarly, a theoretical solution for each column was obtained and combined with the simulation results listed in 
TABLE 2: Theoretical solutions and numerical results of impact responses.

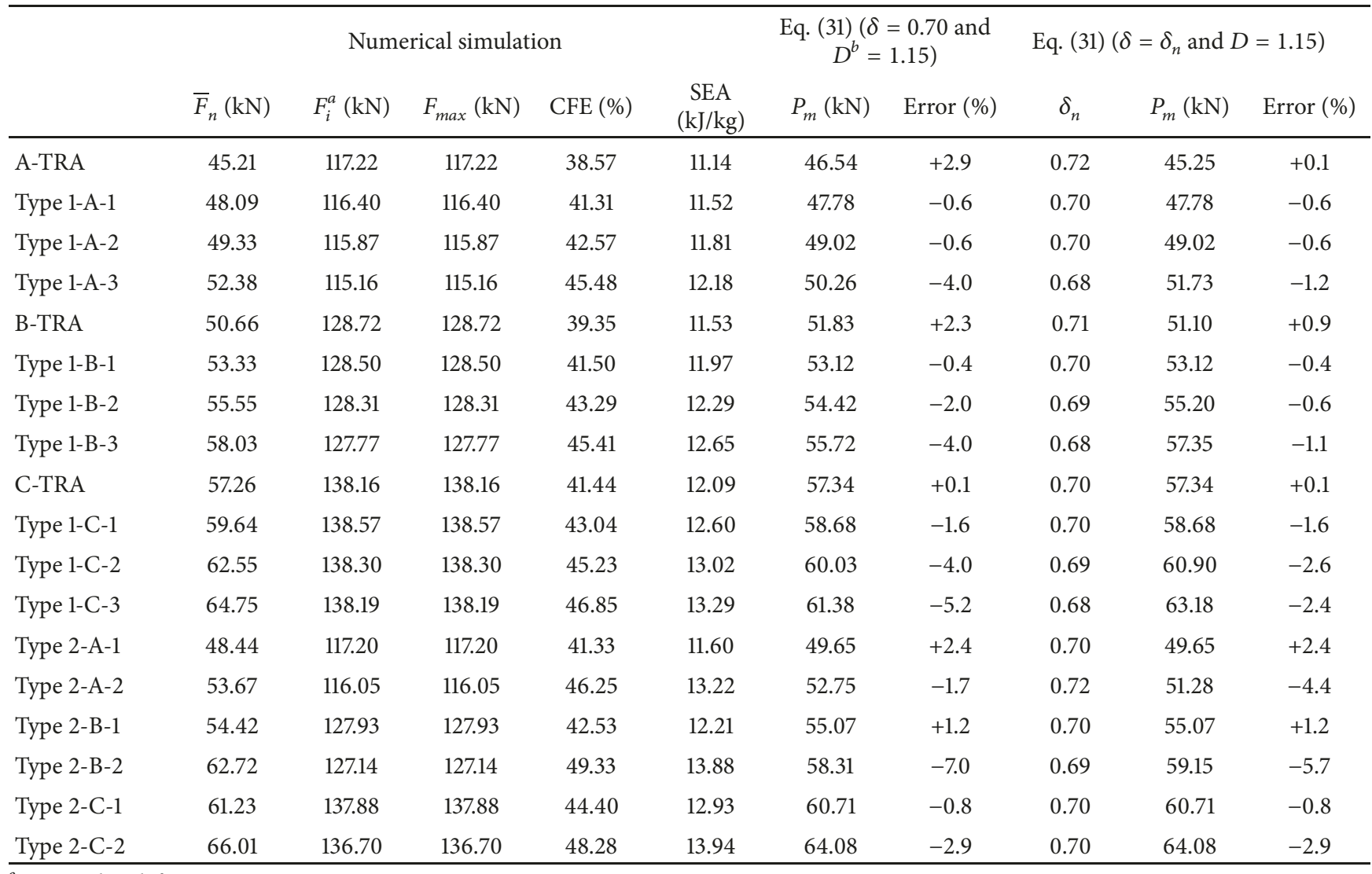

${ }^{\mathrm{a}} F_{i}=$ initial peak force.

${ }^{\mathrm{b}} D=$ dynamic amplification coefficient.

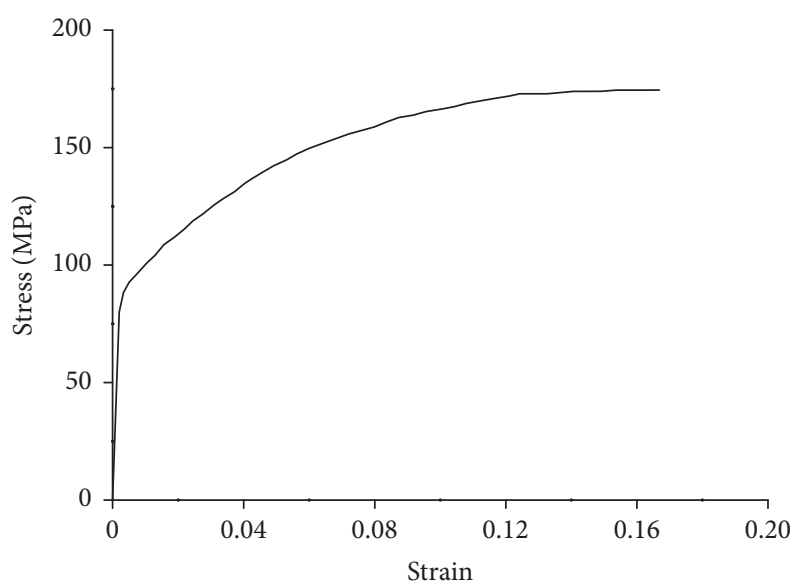

FIGURE 9: Engineering stress-strain curve of AA6060 T4.

Table 2. The numerical mean crushing force $\bar{F}_{n}$ is defined as follows:

$$
\bar{F}_{n}=\frac{\int_{0}^{\delta_{n} L} F(X) d x}{\delta_{n} L},
$$

where $\delta_{n}$ is the effective crushing distance coefficient of the numerical solution and $F(X)$ is the crushing force.
The SEA was calculated as follows:

$$
S E A=\frac{E_{i n t}}{m}=\frac{\bar{F}_{n} \cdot \delta_{n} L}{m},
$$

where $E_{\text {int }}$ and $m$ are the dissipated energy and mass, respectively. The crushing force efficiency (CFE) is an important parameter for evaluating the load consistency of an energy absorbing device. A higher value indicates excellent energy absorbing stability. The CFE is defined as follows:

$$
C F E=\frac{\bar{F}_{n}}{F_{\max }} .
$$

The results listed in Table 2 reveal that the theoretical mean crushing forces agree well with the simulated values. Furthermore, there is good agreement between the simulation results and modified theoretical mean crushing forces, which were calculated using the effective crushing distance coefficient derived from the numerical simulations. Therefore, for AA6060 T4 DSG square tubes and the geometric configurations described above, a dynamic enhancing coefficient of 1.15 adequately accounts for imperfections in the tubes.

In general, all the modified theoretical solutions for the Type 1 DSG tubes were closer to the simulation values compared to the Type 2 DSG tubes. The maximum error of 


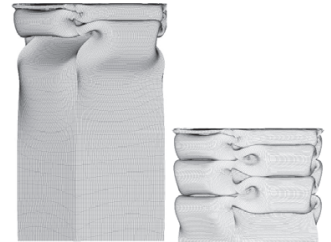

A-TRA

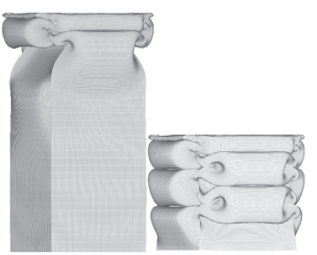

Type1-A-1

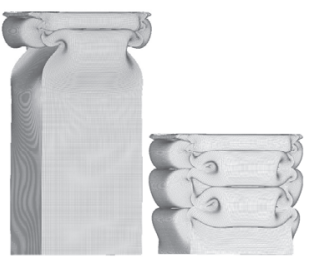

Type1-A-2

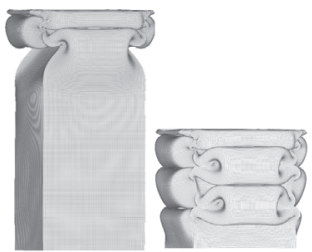

Type1-A-3

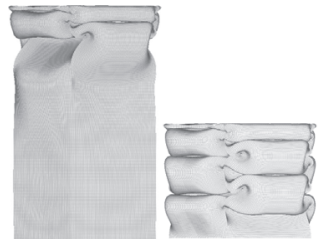

Type2-A-1

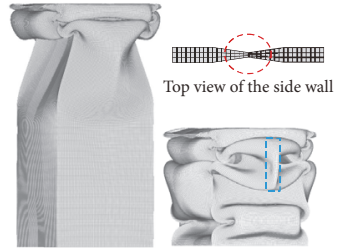

Type2-A-2

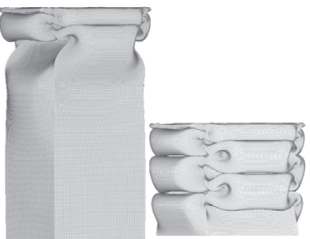

B-TRA

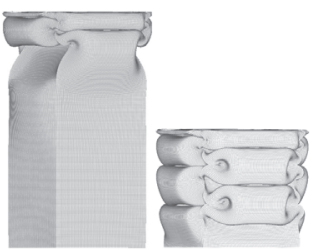

Type1-B-1

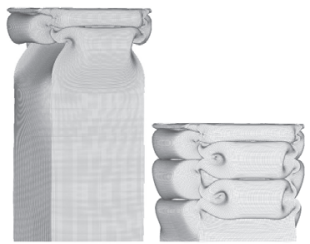

Type1-B-2

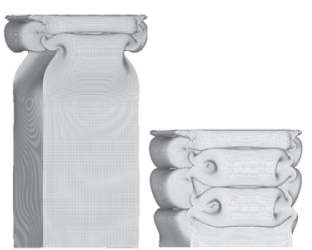

Type1-B-3

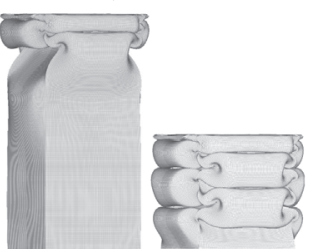

Type2-B-1

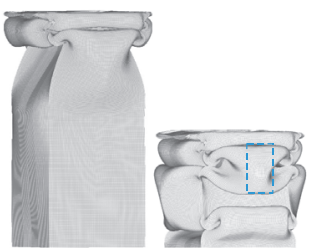

Type2-B-2

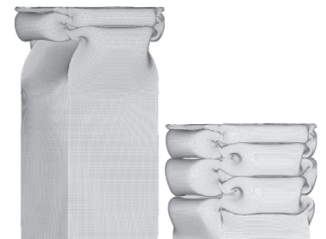

C-TRA

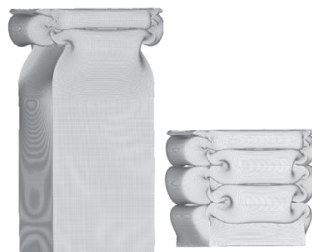

Type1-C-1

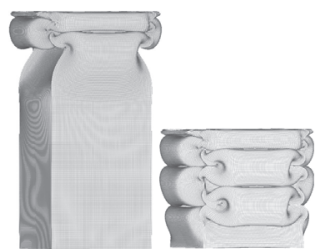

Type1-C-2

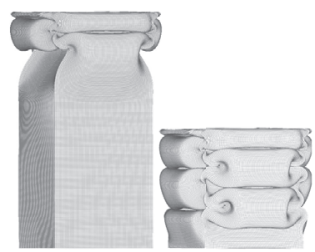

Type1-C-3

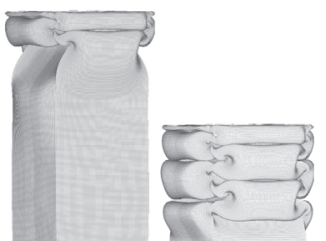

Type2-C-1

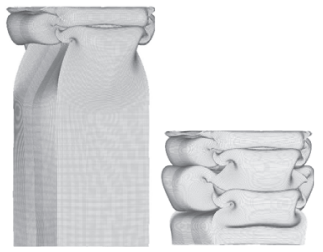

Type2-C-2

FIGURE 10: Deformation patterns of the columns.

Type 1 DSG tubes was approximately $-2.6 \%$ (for Type 1-C2 ). For the two Type 2 DSG tubes (Type 2-A-2 and Type 2$\mathrm{B}-2)$, the errors were $-4.4 \%$ and $-5.7 \%$, respectively. This is largely attributed to the irregular deformation modes of the two tubes, as shown in Figure 10.

As illustrated in Figure 10, all initial folds developed at the tops of the tubes because imperfections were introduced in the top regions. All Type 1 DSG tubes folded in the symmetric quasi-inextensional mode, from which the expression for the mean load was derived above. However, the folding patterns of the two Type 2 DSG tubes (Type 2-A-2 and Type 2-B-2) were noticeably different from those of the other specimens. Therefore, the derived mean load expression was not appropriate for these tubes. Additionally, a notable characteristic of the irregular deformation mode was vertical irregular folds, as shown by the blue dashed boxes in Figure 10. The occurrence of this irregular folding is mainly attributed to the long folding wavelength. Additionally, the spacing of the two opposite plates was significantly reduced during the inward movement of the irregular fold (see Type 2-A-2 and 


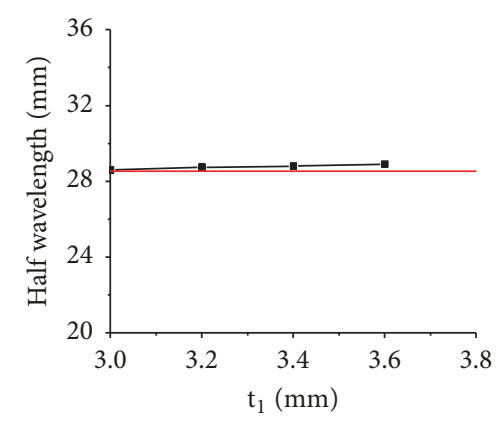

$\rightarrow-$ Type I DSG tubes in group A; $t_{a}=3.0 \mathrm{~mm}$ — Theoretical prediction of A-TRA by Eq.(25)

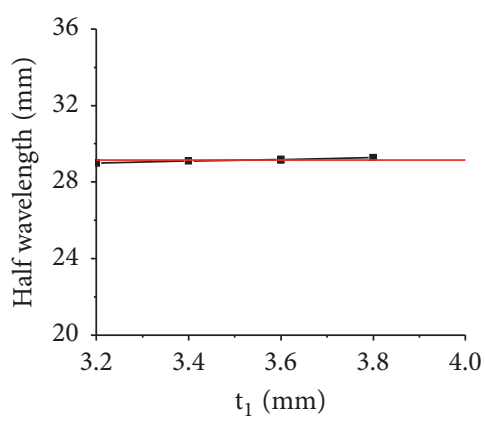

-- Type I DSG tubes in group $B ; t_{a}=3.2 \mathrm{~mm}$ — Theoretical prediction of B-TRA by Eq.(25)

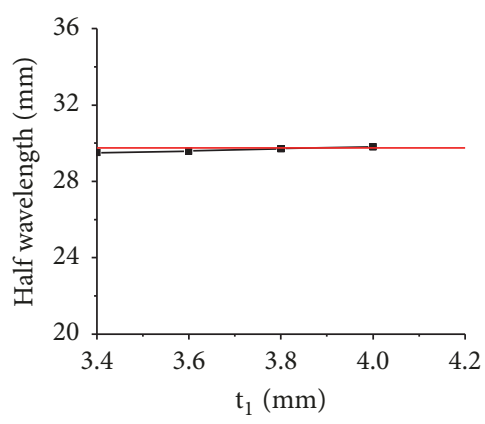

$\rightarrow-$ Type I DSG tubes in group C; $\mathrm{t}_{\mathrm{a}}=3.4 \mathrm{~mm}$ — Theoretical prediction of C-TRA by Eq.(25)

Figure 11: Half wavelength maximum thickness $\left(t_{1}\right)$ curves of Type 1 DSG tubes in groups: (a) A, (b) B, and (c) C.

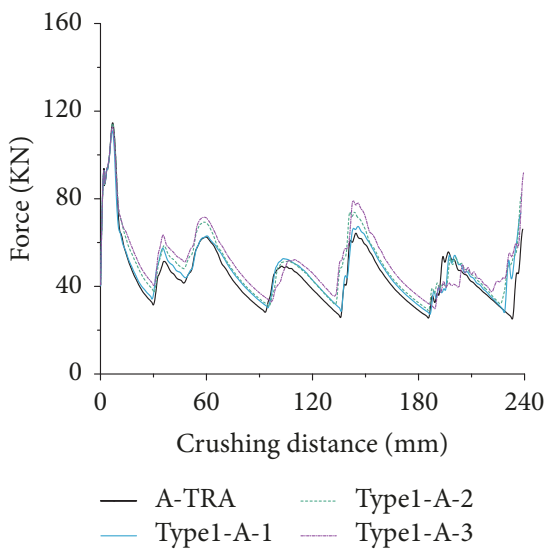

(a)

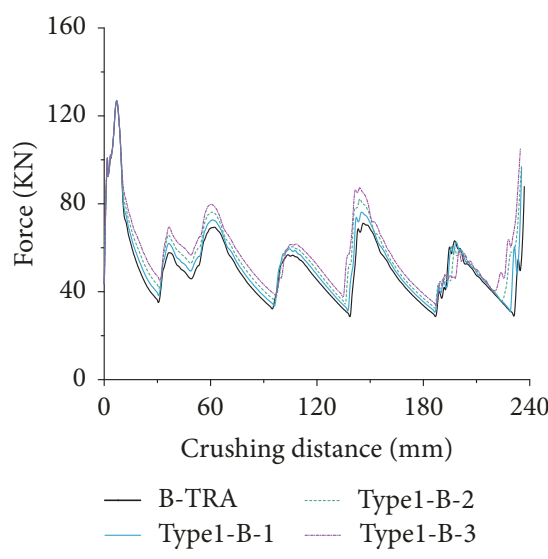

(b)

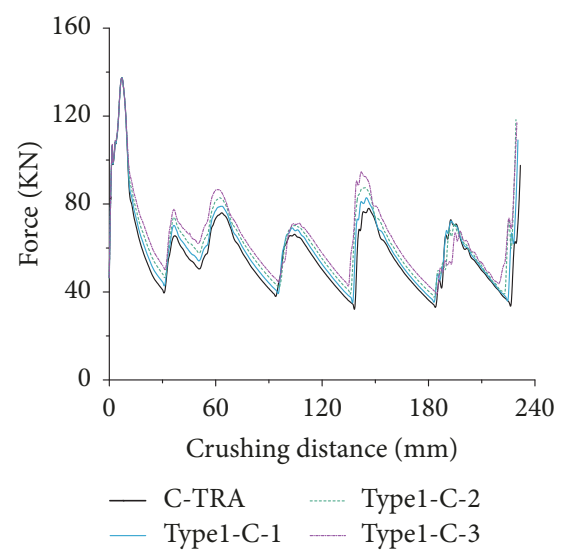

(c)

FIgURE 12: Crushing load responses of Type 1 DSG tubes for three average thickness conditions: (a) $t_{a}=3.0 \mathrm{~mm}$, (b) $t_{a}=3.2 \mathrm{~mm}$, and (c) $t_{a}=3.4 \mathrm{~mm}$.

Type 2-B-2). Simultaneously, the other two opposing plates bulged outward in the middle regions, which were relatively thinner. These thinner middle regions are characterized by a minimum thickness value $t_{2}$, which is relatively small, as indicated by the dashed circle in Figure 10. Accordingly, the relatively long folding wavelength and smaller value of $t_{2}$ lead to atactic folding. For example, although Type 2-A-2 had a similar global average thickness $\left(t_{a}\right)$, and maximum thickness $\left(t_{1}\right)$ compared to Type 1-A-2, there was a significant reduction in the $t_{2}$ value of Type 2-A-2 compared to that of Type 1A-2. Therefore, an irregular fold occurred in the Type 2-A-2 specimen but not in the Type 1-A-1 specimen. The difference between the deformation modes of the Type 1-B-2 and Type 2B-2 specimens can be explained similarly. Additionally, the $t_{2}$ values of the other four Type 2 DSG tubes (Type 2-A-1, Type 2B-1, Type 2-C-1, and Type 2-C-2) did not decrease sufficiently or trigger the irregular mode.

4.3.2. Influences of the Two Main Parameters $\left(t_{\boldsymbol{e}}\right.$ and $\left.t_{\boldsymbol{a}}\right)$. According to the theoretical analysis presented in Section 3, the crushing resistances of the DSG square tubes with consistent materials, cross-sectional widths, and boundary conditions were primarily influenced by two crucial parameters: the average thickness of the EEAR $t_{e}$ and global average thickness $t_{a}$. In this section, the effects of these two parameters on the crushing responses will be discussed.

Figures 12 and 13 present the force-displacement curves of the grouped Type 1 and Type 2 DSG tubes, respectively. For the Type 1 tubes with $t_{a}=3 \mathrm{~mm}$ (see Figure 12(a)), although the thickness gradients of the Type 1-A-1, Type 1-A-2, and Type 1-A-3 specimens increased, curve undulations of those tubes are similar to those of the traditional tube, namely, ATRA. Additionally, the initial peak forces of the four tubes are nearly identical because they are strongly linked to the global average thickness. These trends were also observed in other groups of Type 1 and Type 2 DSG tubes. However, the curve fluctuations, peak numbers, and peak stages in the curves for the Type 2-A-2 and Type 2-B-2 tubes were significantly different from those of the other tubes (see Figures 13(a) and 13(b)), which can be attributed to their irregular folding patterns.

According to (31), the mean load increases with an increasing value of $t_{\boldsymbol{e}}$, while the other parameters remain unchanged. The extent of this increase is closely related to the coefficient of $t_{e} / t_{a}$. This can also be proven based on the simulation results in Table 2. The maximum increase in the mean load of the Type 1 DSG tubes was $13.1 \%$ compared to 


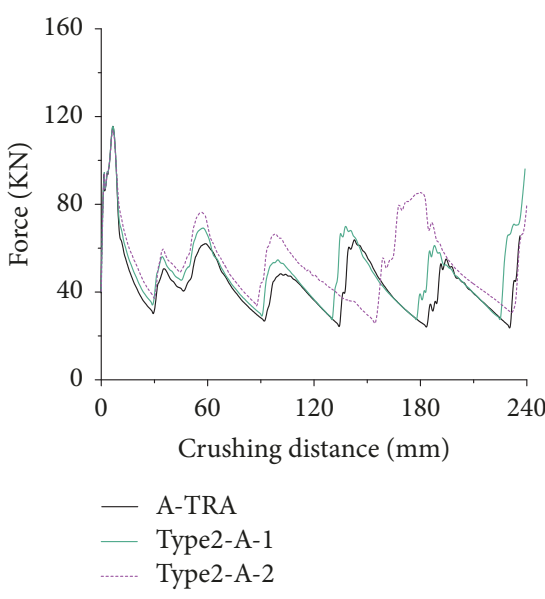

(a)

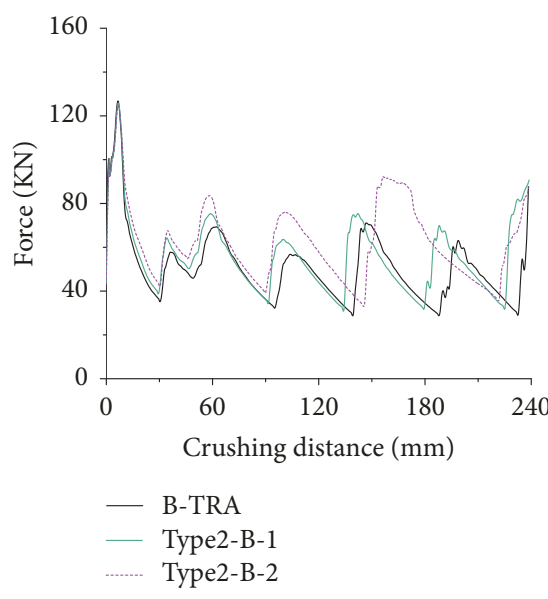

(b)

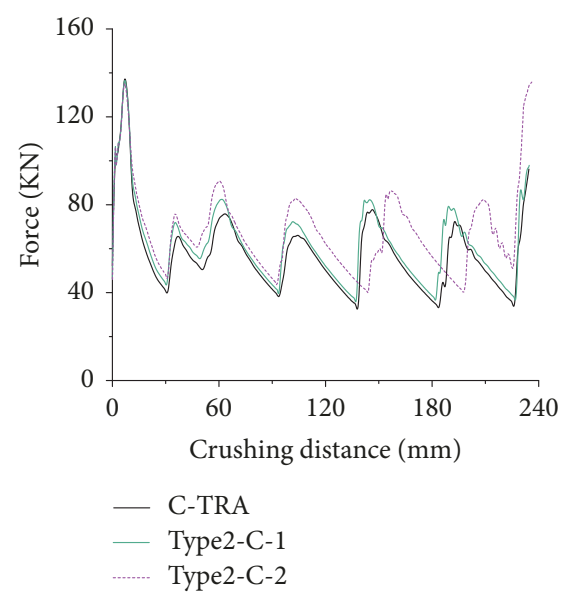

(c)

FIgURE 13: Crushing load responses of Type 2 DSG tubes for three average thickness conditions: (a) $t_{a}=3.0 \mathrm{~mm}$, (b) $t_{a}=3.2 \mathrm{~mm}$, and (c) $t_{a}=3.4 \mathrm{~mm}$.

the traditional C-TRA tube, which was determined using the Type 1-C-3 tube. For the Type 2 DSG tubes, the maximum increase in the mean load was $23.8 \%$ compared to the BTRA tube, which was determined using the Type 2-B-2 tube. The reason why the Type 2 DSG tubes excelled at mean load enhancement is that the EEAR of Type 2 tube can contain more material compared to Type 1 , when $t_{\boldsymbol{a}}$ is constant. Therefore, Type 2 is more efficient at increasing the coefficient of $t_{e} / t_{a}$. This coefficient will be discussed in detail later.

In addition to the parameter $t_{e}$, the global average thickness $t_{a}$ is another essential variable related to the mean load. Its influence on mechanical responses is discussed below. Several important phenomena can be observed in the curves in Figures 12 and 13 and corresponding data in Table 2. First, nearly identical initial peak forces were obtained for the tubes with a constant value of $t_{a}$, regardless of the material distribution type and thickness gradient. Second, the initial peak force increases with an increasing global average thickness, which is also independent of the material distribution type and thickness gradient. As illustrated in Figures 12(a)-12(c), the initial peak force of the Type 1 DSG tubes increased from approximately 116 to $138 \mathrm{KN}$ with an increase in $t_{a}$ from 3.0 to $3.4 \mathrm{~mm}$. This trend can also be observed in the response curves (Figures 13(a)-13(c)) of the Type 2 DSG tubes. Finally, the ideal energy absorber has a high mean load and low initial peak force. Therefore, the best DSG square tube should have a large coefficient of $t_{e} / t_{a}$. Again, this coefficient proved to be an important factor affecting crashworthiness. However, such a DSG square tube may experience atactic deformation, as demonstrated by the Type 2-A-2 and Type 2-B-2 specimens. As mentioned above, the occurrence of irregular deformation is strongly linked to the minimum thickness $t_{2}$, which is closely related to the material distribution.

4.3.3. Influence of Material Distribution Type. For a DSG tube with a constant global average thickness $t_{a}$, the minimum thickness $t_{2}$ decreases with an increasing maximum thickness $t_{1}$. The $t_{2}$ values of Type 2 DSG tubes decrease more rapidly based on their material distribution characteristics. Therefore, each Type 2 DSG tube has a smaller $t_{2}$ value compared to the Type 1 DSG tube with the same $t_{1}$ and $t_{a}$ values, as indicated by the physical properties listed in Table 1 . Based on the reduction of $t_{2}$ for the Type 2-A-2 and Type 2-B-2 tubes beyond a certain limit, irregular folds occurred. There was a relatively small reduction in the $t_{2}$ values of the Type 1-A-2 and Type 1-B-2 tubes, which deformed via regular deformation modes.

As mentioned above, in the theoretical expression (31), the load efficiency of a DSG column increases with an increase in $t_{\boldsymbol{e}}$, while the other parameters remain unchanged. Therefore, the purpose of changing the material distribution is to increase the value of $t_{e}$. Of the two types of material distributions, Type 2 is more efficient than Type 1 for increasing $t_{\boldsymbol{e}}$. As shown in Table 1 , the $t_{\boldsymbol{e}}$ value of each Type 2 DSG column was larger than that of the Type 1 DSG column with the same $t_{a}$ and $t_{1}$ values. For example, the $t_{\boldsymbol{e}}$ value of Type 2-A-2 increased to $3.4 \mathrm{~mm}$ when $t_{1}$ increased to $3.4 \mathrm{~mm}$. However, for Type 1-A-2, $t_{e}$ only increased to $3.16 \mathrm{~mm}$. Therefore, the mean load and SEA of the Type 2-A-2 tube are higher than those of the Type 1-A-2 tube, which was proven by the theoretical prediction and simulation results. The efficiency of the Type 2 material distribution for promoting $t_{\boldsymbol{e}}$ mainly stems from the fact that the EEAR of the Type 2 DSG tubes holds more material compared to that of the Type 1 DSG tubes.

Finally, from the perspective of crashworthiness improvement, the main concerns are the load efficiency and regular folding patterns that a given tube can produce. Therefore, a material distribution type that ensures a fixed $t_{2}$ value and will not trigger irregular folding while allowing the EEAR to hold as much material as possible is desirable.

\section{Modification of Material Distributions}

5.1. Improvement of the Type 2 Material Distribution. As discussed above, for a Type 2 DSG tube with a constant global average thickness, concentrating material within the EEAR 


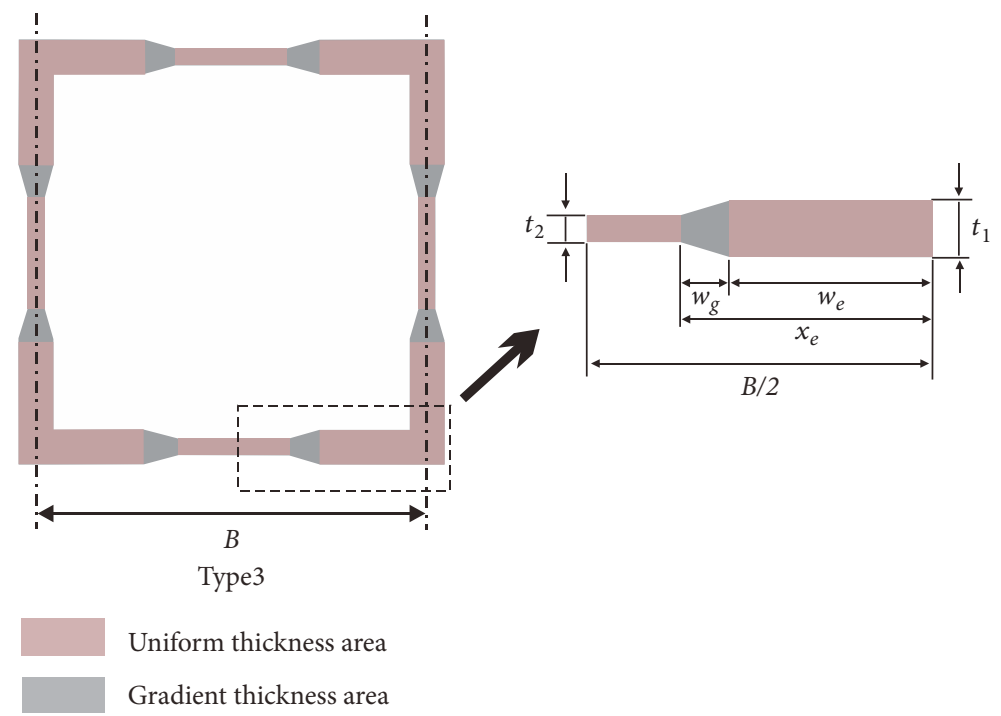

FIgURE 14: Material distribution of Type 3 tubes.

will lead to a significant decrease in the minimum thickness $\left(t_{2}\right)$, which limits the promotion of the average thickness of the EEAR $\left(t_{\boldsymbol{e}}\right)$. Therefore, a modified material distribution type should maximize the value of $t_{\boldsymbol{e}}$ under such limitations. Based on this principle, the Type 2 material distribution was modified to create a new distribution.

The geometric configuration of this new material distribution type (Type 3) is illustrated in Figure 14. As shown in the figure, the non-EEAR was given a uniform thickness equal to the minimum thickness $t_{2}$, which ensures that the EEAR contains the most material for a fixed value of $t_{2}$. Additionally, the EEAR has a uniform thickness area with a width of $w_{e}$ and gradient thickness area with a width of $w_{g}$, as shown in Figure 14. In other words, the width of the EEAR $x_{e}$ is equal to the sum of $w_{e}$ and $w_{g}$. The crushing response improvements provided by the Type 3 material distribution are discussed below.

5.2. Crushing Response Comparison. In theory, for two DSG tubes with the same cross-sectional width and global average thickness, a higher energy absorption efficiency is achieved by the tube with a larger $t_{e}$ value. The $t_{e}$ value for a Type 1 or Type 3 DSG tube can be obtained easily by using the relationships between its geometric parameters, as shown in Table 3. Additionally, it is necessary to determine if a modified Type 2 DSG tube (i.e., Type 3 DSG tube) is more efficient than a Type 1 DSG tube in terms of energy absorption for the same geometric constraints, namely, cross-sectional width $(B)$, global average thickness $\left(t_{a}\right)$, and minimum thickness $\left(t_{2}\right)$. The geometric constraints of $B=90 \mathrm{~mm}, t_{a}=$ $3.2 \mathrm{~mm}$, and $t_{2}=2.0 \mathrm{~mm}$ were used for the two types of DSG tubes. The maximum $t_{e}$ values are listed in Table 4 . The Type 1 DSG tube with geometric constraints was labelled Type 1-BMAX. Similarly, considering the same geometric constraints, three Type 3 DSG tubes with $w_{g}+w_{e}=x_{e}$ were established, where the $w_{g}$ values were set to $0.25 x_{e}, 0.5 x_{e}$, and $0.75 x_{e}$ to investigate the effects of this parameter. These three tubes were labelled Type 3-B-MAX1, Type 3-B-MAX2, and Type 3$\mathrm{B}-\mathrm{MAX} 3$, respectively. All the dimensions of the Type 1 and three Type 3 tubes are listed in Table 4.

Numerical simulations of the four tubes under the boundary conditions mentioned above were carried out. The force-crushing distance curves and deformation modes are presented in Figures 15(a) and 15(b), respectively. First, as shown in Figure 15(b), all four tubes were crushed in progressive and regular deformation modes. The folding lobes of the three Type 3 DSG tubes were slightly larger than those of the Type 1 DSG tube. Second, the simulation results listed in Table 4 show that, compared to the traditional square tube, the improvements in terms of CFE and SEA for the Type 1 -B-MAX tube were $23.7 \%$ and $17.9 \%$, respectively. Greater improvements in these two indexes were obtained by the Type 3 DSG tubes, with values of $41.9 \%$ and $30.6 \%$, respectively. Additionally, for the three Type 3 DSG tubes with $w_{g}+w_{e}=$ $x_{e}$, the mean load increased slightly with an increasing value of $w_{g}$, while the SEA decreased slightly. This decrease is related to the effective crushing distance, which decreases with an increasing value of $w_{g}$, as shown in the response curves in Figure 15(a). Therefore, although $w_{g}$ has a slight effect on the crushing responses, from the perspective of energy absorption efficiency, a smaller $w_{g}$ value is required to guarantee a gentle thickness gradient change. Finally, under the same geometric constraints with constant values of $t_{a}$ and $t_{2}$, a higher value of $t_{e}$ was achieved by the Type 3 DSG tubes compared to the Type 1 DSG tube, which led to a higher energy absorption efficiency. Therefore, the key to improving the crushing performance of a DSG tube is an appropriate material distribution type.

\section{Conclusion}

DSG square tubes were constructed by concentrating additional material in the corner regions by changing the cross-sectional material distributions. The energy dissipating 


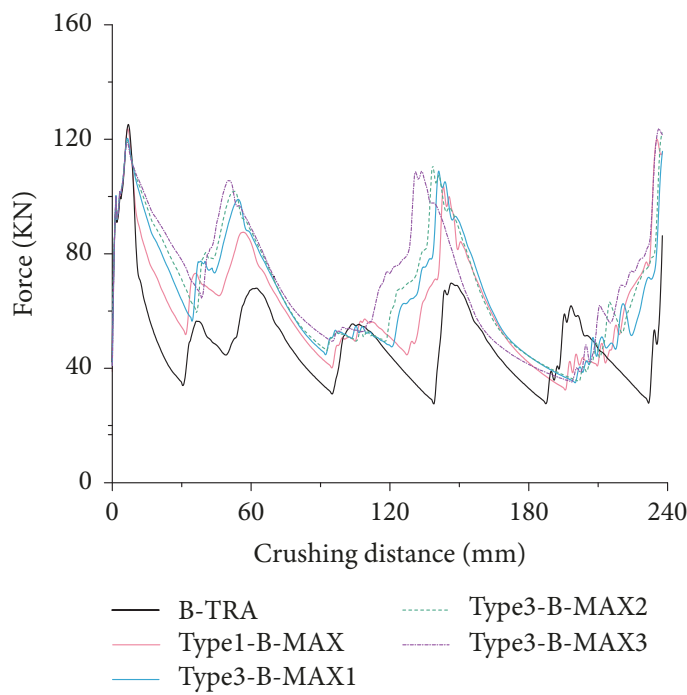

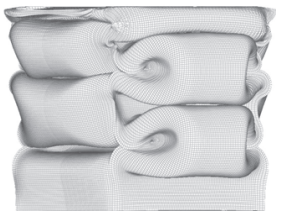

Type1-B-MAX

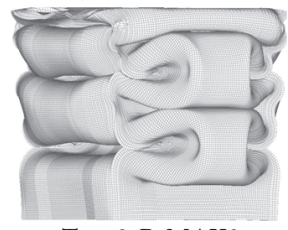

Type3-B-MAX2

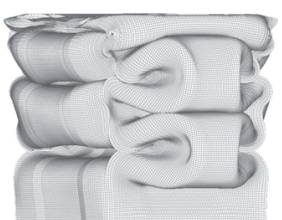

Type3-B-MAX1

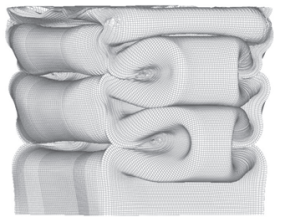

Type3-B-MAX3

FIGURE 15: Crushing responses of specimens with dimensional constraints: (a) force-crushing distance curves and (b) folding patterns.

TABLE 3: Relationships between geometric parameters.

\begin{tabular}{|c|c|c|c|c|}
\hline $\begin{array}{l}\text { Gradient thickness } \\
\text { type }\end{array}$ & Width of the EEAR & $\begin{array}{c}\text { Widths of the gradient } \\
\text { thickness area and uniform } \\
\text { thickness area }\end{array}$ & Maximum thickness & $\begin{array}{c}\text { Average thickness } \\
\text { of the EEAR }\end{array}$ \\
\hline Type 1 & $x_{e}=0.95 H$ & $\begin{aligned} w_{g} & =\frac{B}{2} \\
w_{e} & =0\end{aligned}$ & $t_{1}=2 t_{a}-t_{2}$ & $t_{e}=\frac{\left(t_{2}-t_{1}\right) x_{e}}{B}+t_{1}$ \\
\hline Type 3 & $x_{e}=0.95 H$ & $w_{g}+w_{e}=x_{e}$ & $\begin{array}{c}t_{1}= \\
\frac{B\left(t_{a}-t_{2}\right)+t_{2} x_{e}+t_{2} w_{e}}{x_{e}+w_{e}}\end{array}$ & $t_{e}=\frac{B\left(t_{a}-t_{2}\right)}{2 x_{e}}+t_{2}$ \\
\hline
\end{tabular}

TABLE 4: Dimensions and crushing responses of two types of specimens with dimensional constraints.

\begin{tabular}{lccccccccccc}
\hline Specimen & \multicolumn{3}{c}{ Dimension } & & & \multicolumn{3}{c}{ Simulation result } \\
& \multicolumn{3}{c}{$\begin{array}{c}\text { Constraint } \\
(\mathrm{g})\end{array}$} & $t_{a}(\mathrm{~mm})$ & $t_{2}(\mathrm{~mm})$ & $t_{1}(\mathrm{~mm})$ & $t_{e}(\mathrm{~mm})$ & $w_{g}$ & $w_{e}$ & $\begin{array}{c}P_{m}(\mathrm{kN}) \\
\begin{array}{c}\text { CFE } \\
(\%)\end{array}\end{array} \begin{array}{c}\text { SEA } \\
(\mathrm{kJ} / \mathrm{kg})\end{array}$ \\
\hline B-TRA & 997.6 & 3.20 & 3.20 & 3.20 & 3.20 & 0 & $B / 2$ & 50.66 & 39.35 & 11.53 \\
Type 1-B-MAX & 997.6 & 3.20 & 2.00 & 4.40 & 3.71 & $B / 2$ & 0 & 62.35 & 48.71 & 13.60 \\
Type 3-B-MAX1 & 997.6 & 3.20 & 2.00 & 4.37 & 4.10 & $0.25 x_{e}$ & $0.75 x_{e}$ & 67.10 & 54.80 & 15.06 \\
Type 3-B-MAX2 & 997.6 & 3.20 & 2.00 & 4.73 & 4.10 & $0.50 x_{e}$ & $0.50 x_{e}$ & 67.87 & 55.42 & 14.80 \\
Type 3-B-MAX3 & 997.6 & 3.20 & 2.00 & 5.22 & 4.10 & $0.75 x_{e}$ & $0.25 x_{e}$ & 68.53 & 55.87 & 14.72 \\
\hline
\end{tabular}

performance of the DSG square structures under an axial dynamic load was investigated both theoretically and numerically.

A mathematical model for the mean crushing force of the DSG square columns was developed. Two key parameters, namely, the global average thickness $\left(t_{a}\right)$ and average thickness of the $\operatorname{EEAR}\left(t_{e}\right)$, were proposed. The analytical prediction results were in good agreement with the simulation results. The influences of two key parameters on the impact responses of the columns were discussed.

For the same length, section width, and global average thickness of columns, a larger $t_{e}$ value led to a greater mean crushing load. Similarly, when the other parameters remained unchanged, a smaller $t_{a}$ value led to a lower initial peak force for the columns. Therefore, a larger coefficient of $t_{e}$ versus $t_{a}$ results in a column with excellent energy absorption efficiency and better crashworthiness performance.

To investigate the influence of the cross-sectional material distribution law on increasing the coefficient of $t_{e} / t_{a}$, two material distribution types (Type 1 and Type 2) were presented, and their characteristics were studied. First, Type 2 was more efficient than Type 1 for promoting $t_{e} / t_{a}$. The $t_{e} / t_{a}$ of each Type 2 tube is larger than the Type 1 tube with the same thickness gradient as the Type 2 tube. In 
addition, a thinner thickness of the middle of each edge was found in cases with a large thickness gradient of Type 2 tubes, not Type 1 tubes. Thus, Type 2 tubes more easily deform in irregular modes. Therefore, a modified material distribution type (Type 3) was established, which maintained the advantages of Type 2 and remedied its shortcomings.

An excellent performance of energy absorption efficiency was achieved by Type 3 tubes, and they were not deformed in the irregular deformation mode that occurred on Type 2 tubes. The SEA values of Type 3 tubes with dimensional constraints increased by $30.6 \%$ compared to those of traditional square tubes, which is higher than those of the other two types. Therefore, the Type 3 is superior in the three types.

In summary, as a novel design, the DSG square tube is a superior structure that can meet increasingly stringent requirements for energy absorption efficiency. Although more extensive investigations must be carried out, this study presents a advancement in the comprehensive understanding of the folding mechanisms of square tubes with graded thicknesses, which is advantageous for the design improvement of such structures in terms of deriving more efficient energy absorbing devices.

\section{Data Availability}

The data used to support the findings of this study are available from the corresponding author upon request.

\section{Conflicts of Interest}

The authors declare that they have no competing financial interests.

\section{Acknowledgments}

This work was supported by the National Natural Science Foundation of China (no. 11272070).

\section{References}

[1] A. Airoldi and G. Janszen, "A design solution for a crashworthy landing gear with a new triggering mechanism for the plastic collapse of metallic tubes," Aerospace Science and Technology, vol. 9, no. 5, pp. 445-455, 2005.

[2] D. Tyrell, K. Jacobsen, E. Martinez, and A. B. Perlman, "Trainto-train impact test of crash energy management passenger rail equipment: structural results," in Proceedings of the ASME 2006 International Mechanical Engineering Congress and Exposition, pp. 35-44, Chicago, Ill, USA, 2006.

[3] J. M. Alexander, "An approximate analysis of the collapse of thin cylindrical shells under axial loading," Quarterly Journal of Mechanics and Applied Mathematics, vol. 13, no. 1, pp. 10-15, 1960.

[4] K. R. F. Andrews, G. L. England, and E. Ghani, "Classification of the axial collapse of cylindrical tubes under quasi-static loading," International Journal of Mechanical Sciences, vol. 25, no. 9-10, pp. 687-696, 1983.

[5] W. Abramowicz and N. Jones, "Dynamic axial crushing of circular tubes," International Journal of Impact Engineering, vol. 2, no. 3, pp. 263-281, 1984.
[6] S. Guillow, G. Lu, and R. Grzebieta, "Quasi-static axial compression of thin-walled circular aluminium tubes," International Journal of Mechanical Sciences, vol. 43, no. 9, pp. 2103-2123, 2001.

[7] P. H. Thornton and C. L. Magee, “The interplay of geometric and materials variables in energy absorption," Journal of Engineering Materials and Technology, vol. 99, no. 2, pp. 114-120, 1977.

[8] W. Abramowicz and N. Jones, "Transition from initial global bending to progressive buckling of tubes loaded statically and dynamically," International Journal of Impact Engineering, vol. 19, no. 5-6, pp. 415-437, 1997.

[9] T. Wierzbicki and W. Abramowicz, "On the crushing mechanics of thin-walled structures," Transactions ASME-Journal of Applied Mechanics, vol. 50, no. 4, pp. 727-734, 1983.

[10] X. Zhang and H. Huh, "Crushing analysis of polygonal columns and angle elements," International Journal of Impact Engineering, vol. 37, no. 4, pp. 441-451, 2010.

[11] M. Yamashita, M. Gotoh, and Y. Sawairi, "Axial crush of hollow cylindrical structures with various polygonal cross-sections," Journal of Materials Processing Technology, vol. 140, no. 1-3, pp. 59-64, 2003.

[12] A. Godat, F. Legeron, and D. Bazonga, "Stability investigation of local buckling behavior of tubular polygon columns under concentric compression," Thin-Walled Structures, vol. 53, pp. 131-140, 2012.

[13] X. Zhang and H. Zhang, "Experimental and numerical investigation on crush resistance of polygonal columns and angle elements," Thin-Walled Structures, vol. 57, pp. 25-36, 2012.

[14] Z. Tang, S. Liu, and Z. Zhang, "Energy absorption properties of non-convex multi-corner thin-walled columns," Thin-Walled Structures, vol. 51, pp. 112-120, 2012.

[15] S. Liu, Z. Tong, Z. Tang, Y. Liu, and Z. Zhang, "Bionic design modification of non-convex multi-corner thin-walled columns for improving energy absorption through adding bulkheads," Thin-Walled Structures, vol. 88, pp. 70-81, 2015.

[16] Z. Fan, G. Lu, and K. Liu, "Quasi-static axial compression of thin-walled tubes with different cross-sectional shapes," Engineering Structures, vol. 55, pp. 80-89, 2013.

[17] W. Liu, Z. Lin, N. Wang, and X. Deng, "Dynamic performances of thin-walled tubes with star-shaped cross section under axial impact," Thin-Walled Structures, vol. 100, pp. 25-37, 2016.

[18] C. Baykasoglu and M. T. Cetin, "Energy absorption of circular aluminium tubes with functionally graded thickness under axial impact loading," International Journal of Crashworthiness, vol. 20, no. 1, pp. 95-106, 2015.

[19] F. Xu, X. Tian, and G. Li, "Experimental study on crashworthiness of functionally graded thickness thin-walled tubular structures," Experimental Mechanics, vol. 55, no. 7, pp. 13391352, 2015.

[20] G. Sun, F. Xu, G. Li, and Q. Li, "Crashing analysis and multiobjective optimization for thin-walled structures with functionally graded thickness," International Journal of Impact Engineering, vol. 64, pp. 62-74, 2014.

[21] H. Yin, H. Fang, G. Wen, Q. Wang, and Y. Xiao, "An adaptive RBF-based multi-objective optimization method for crashworthiness design of functionally graded multi-cell tube," Structural and Multidisciplinary Optimization, vol. 53, no. 1, pp. 129-144, 2016.

[22] H. Zhang and X. Zhang, "Crashworthiness performance of conical tubes with nonlinear thickness distribution," ThinWalled Structures, vol. 99, pp. 35-44, 2016. 
[23] X. Zhang, H. Zhang, and Z. Wang, "Bending collapse of square tubes with variable thickness," International Journal of Mechanical Sciences, vol. 106, pp. 107-116, 2016.

[24] F. Xu, X. Zhang, and H. Zhang, "A review on functionally graded structures and materials for energy absorption," Engineering Structures, vol. 171, pp. 309-325, 2018.

[25] A. Meyer, B. Wietbrock, and G. Hirt, "Increasing of the drawing depth using tailor rolled blanks-Numerical and experimental analysis," The International Journal of Machine Tools and Manufacture, vol. 48, no. 5, pp. 522-531, 2008.

[26] M. Kleiner, M. Geiger, and A. Klaus, "Manufacturing of lightweight components by metal forming," CIRP Annals - Manufacturing Technology, vol. 52, no. 2, pp. 521-542, 2003.

[27] X. Zhang, Z. Wen, and H. Zhang, "Axial crushing and optimal design of square tubes with graded thickness," Thin-Walled Structures, vol. 84, pp. 263-274, 2014.

[28] S. P. Santosa, T. Wierzbicki, A. G. Hanssen, and M. Langseth, "Experimental and numerical studies of foam-filled sections," International Journal of Impact Engineering, vol. 24, no. 5, pp. 509-534, 2000.

[29] W. Abramowicz and N. Jones, "Dynamic axial crushing of square tubes," International Journal of Impact Engineering, vol. 2, no. 2, pp. 179-208, 1984.

[30] W. Abramowicz and N. Jones, "Dynamic progressive buckling of circular and square tubes," International Journal of Impact Engineering, vol. 4, no. 4, pp. 243-270, 1986.

[31] W. Abramowicz and T. Wierzbicki, "Axial crushing of multicorner sheet metal columns," Journal of Applied Mechanics, vol. 56, no. 1, pp. 113-120, 1989.

[32] R. J. Hayduk and T. Wierzbicki, "Extensional collapse modes of structural members," Computers \& Structures, vol. 18, no. 3, pp. 447-458, 1984.

[33] J. Ma, D. Hou, Y. Chen, and Z. You, "Quasi-static axial crushing of thin-walled tubes with a kite-shape rigid origami pattern: Numerical simulation," Thin-Walled Structures, vol. 100, pp. 3847, 2016.

[34] A. Hanssen, M. Langseth, and O. Hopperstad, "Static and dynamic crushing of square aluminium extrusions with aluminium foam filler," International Journal of Impact Engineering, vol. 24, no. 4, pp. 347-383, 2000.

[35] M. Langseth and O. S. Hopperstad, "Static and dynamic axial crushing of square thin-walled aluminium extrusions," International Journal of Impact Engineering, vol. 18, no. 7-8, pp. 949968, 1996. 


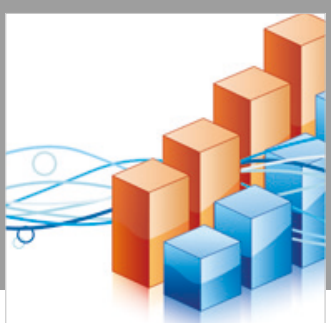

Advances in

Operations Research

\section{-n-m}
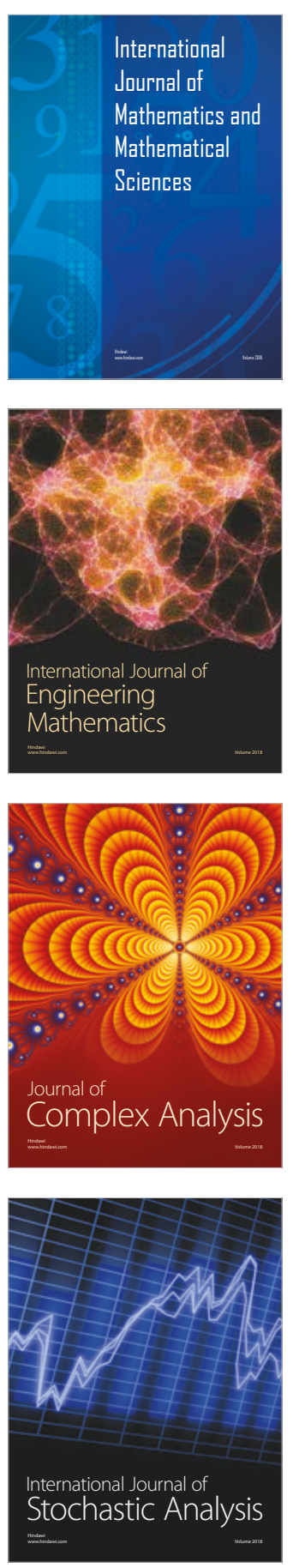
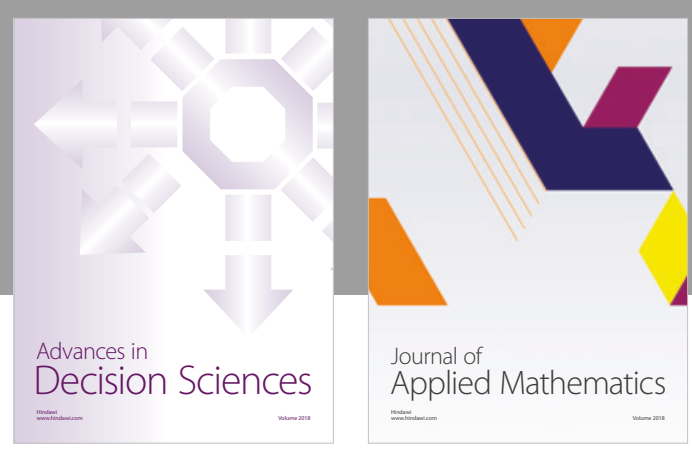

Journal of

Applied Mathematics
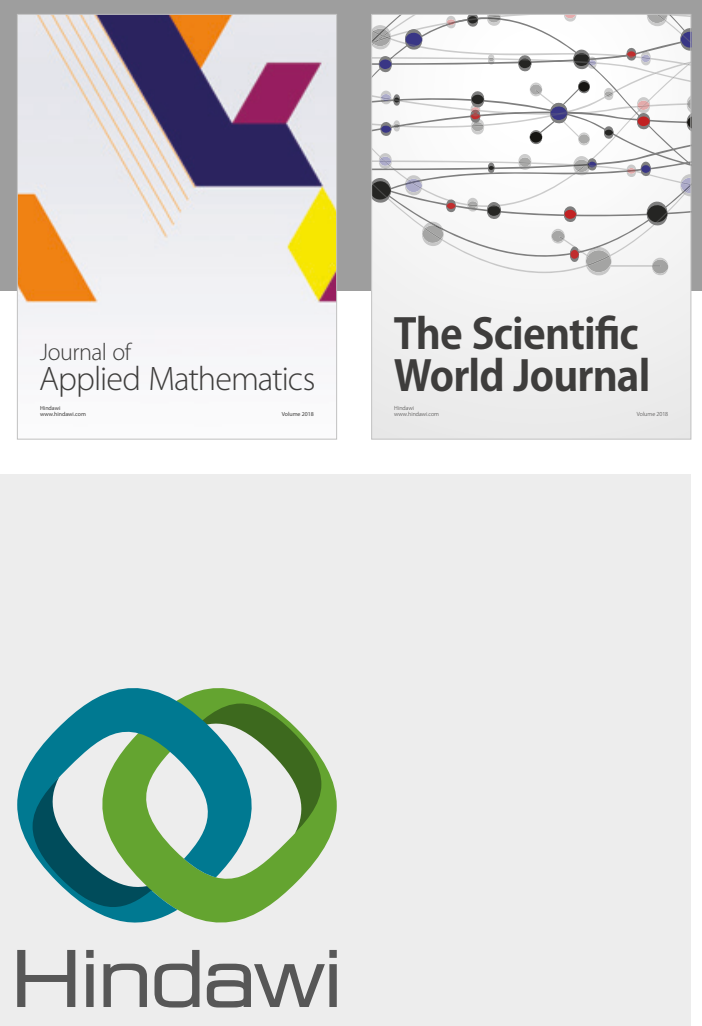

Submit your manuscripts at

www.hindawi.com

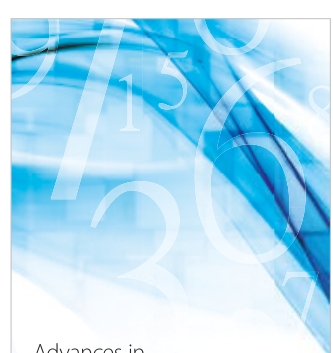

Advances in
Numerical Analysis
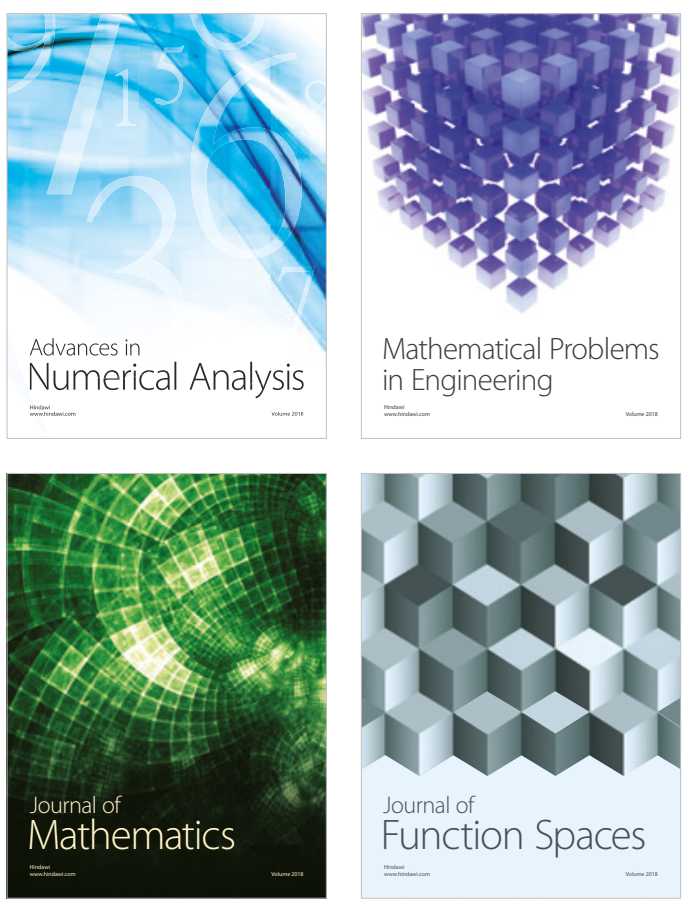

Mathematical Problems in Engineering

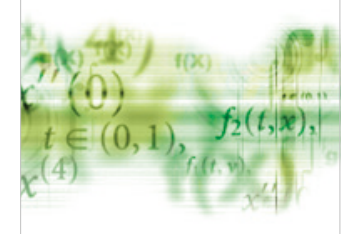

International Journal of

Differential Equations

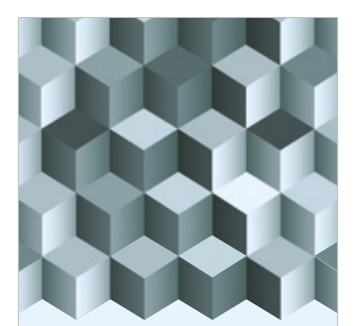

Journal of

Function Spaces

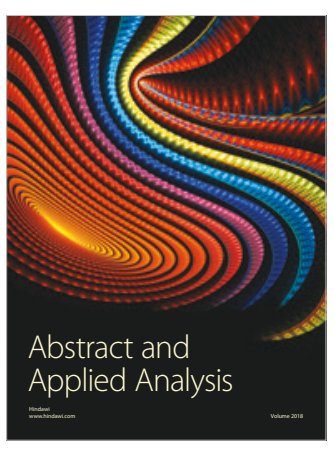

The Scientific

World Journal



Journal of

Probability and Statistics
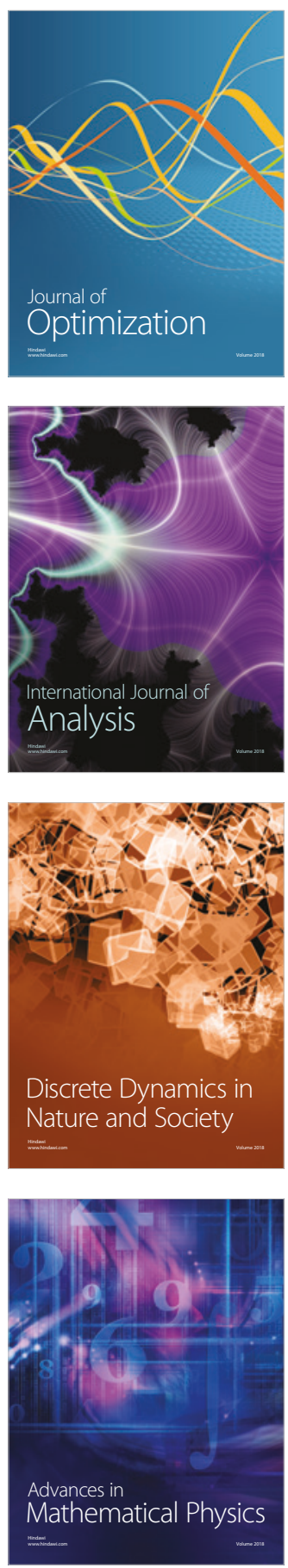